University of Louisville

ThinkIR: The University of Louisville's Institutional Repository

Electronic Theses and Dissertations

$12-2014$

\title{
Enzyme kinetics : 6-phosphofructo-2-kinase/2,6-bisphosphatase.
}

Jennifer Clark

University of Louisville

Follow this and additional works at: https://ir.library.louisville.edu/etd

Part of the Biochemistry, Biophysics, and Structural Biology Commons

\section{Recommended Citation}

Clark, Jennifer, "Enzyme kinetics : 6-phosphofructo-2-kinase/2,6-bisphosphatase." (2014). Electronic Theses and Dissertations. Paper 1720.

https://doi.org/10.18297/etd/1720

This Master's Thesis is brought to you for free and open access by ThinkIR: The University of Louisville's Institutional Repository. It has been accepted for inclusion in Electronic Theses and Dissertations by an authorized administrator of ThinkIR: The University of Louisville's Institutional Repository. This title appears here courtesy of the author, who has retained all other copyrights. For more information, please contact thinkir@louisville.edu. 


\title{
ENZYME KINETICS: 6-PHOSPHOFRUCTO-2-KINASE/2,6- BISPHOSPHATASE
}

\author{
By \\ Jennifer Clark \\ B.A., Franklin College, 2009
}

\begin{abstract}
A Thesis
Submitted to the Faculty of the

School of Medicine of the University of Louisville

In Partial Fulfillment of the Requirements

For the Degree of
\end{abstract}

\author{
Masters of Science \\ Department of Biochemistry and Molecular Biology \\ University of Louisville \\ Louisville, Kentucky
}

December 2014 



\title{
ENZYME KINETICS: 6-PHOSPHOFRUCTO-2-KINASE/2,6- BISPHOSPHATASE
}

\author{
By \\ Jennifer Clark \\ B.A., Franklin College, 2009
}

A Thesis Approved on

November 25, 2014

by the following Thesis Committee:

\begin{tabular}{c}
\hline Dr. Sucheta Telang \\
\hline Dr. Steven Ellis \\
\hline Dr. Barbara Clark
\end{tabular}




\section{ACKNOWLEDGEMENTS}

I am using this opportunity to express my gratitude to everyone who supported me during my journey of attaining my MS degree. First and foremost I offer my sincerest gratitude to my mentor Dr. Sucheta Telang. However, she has been much more than a mentor to me during my times at the University of Louisville. Sucheta has not only been the most understanding, patient, and encouraging mentor in the field of cancer research, but has also been a great friend. The most devastating day in my science career was the day I had to tell her that I was moving to Mississippi because of my husband's job. However, even though I had to leave her lab and the university, she never stopped helping me along the way and encouraging me to stay focused on finishing my degree. I attribute the level of my Masters degree to her help and effort, and without her, this thesis would not have been completed or written. One simply could not wish for a better supervisor. I would also like to show my gratitude to Dr. Barbara Clark and Dr. Steve Ellis. Without their help, especially in completing all the administrative requirements for my degree, I know that I would have most likely given up along the way. They also 
were essential in providing me with endless insight and constructive criticism with my project. I know I would not have been able to complete the requirements for the degree, especially from a different state, without their understanding and flexibility, and for that I will forever be grateful.

I would also like to take the time to thank the faculty and staff in the Department of Biochemistry and Molecular Biology, especially Dr. Geoff Clark and Dr. Howard Donninger, for contributing to my scientific training, offering me my first position at the University of Louisville, and for encouraging me to begin my Masters of science degree. I am very grateful to everyone that I have worked alongside, collaborated with, and trained under over the past five years, including; Dr. Julie O'Neill, Dr.

Yoannis Imbert-Fernandez, Dr. Jason Chesney, Dr. Alden Klarer, Amy Clem, Dr. Brian Clem, Lee Schmidt, Dr. Katherine Hobbing, Dr. Erin Brock, Dr. Abudullah Yalcin, and Bob Miller. I would like to thank my family and friends for their endless support and love. My parents taught me to never give up, to work hard, and to never stop believing in myself. Finally, I would like to thank my husband, Andy Clark, for supporting me in all that I do, in talking me off the ledge when I am stressed, and who pushes me to my limits. I could not ask for a better partner to take on this journey of life with. 


\begin{abstract}
ENZYME KINETICS: 6-PHOSPHOFRUCTO-2-KINASE/FRUCTOSE-2,6BISPHOSPHATASE

Jennifer Clark
\end{abstract}

November 25, 2014

Altered energy metabolism is an established hallmark of cancer cells. Fructose-2,6-bisphosphate is an allosteric activator of glycolysis and its concentration in a cell is dictated by the 6-phosphofructo-2kinase/fructose-2,6-bisphosphatases (PFKFB1-4) family of bifunctional enzymes. The PFKFB family of enzymes are characterized by their different kinase:bisphosphatase activities and were originally determined to be tissue-specific. More recent data however suggest that multiple isoforms are co-expressed in both normal and neoplastic tissues. PFKFB4 is highly expressed in human cancer, strongly induced by hypoxia, and required for the survival of cancer cells However, it remains unclear whether the kinase or phosphatase activity of human PFKFB4 dominates. In this study, we developed a method of synthesizing and purifying human 
recombinant PFKFB4 and, using this purified protein, found that PFKFB4 has a kinase to phosphatase ratio of $4.1: 1$. We found that a novel small molecule inhibitor of PFKFB4 (5MPN) inhibited the kinase activity of PFKFB4 and further that the known PFKFB3 inhibitors (PFK15 and PFK158) inhibited PFKFB4 less efficiently than 5MPN. We conclude that PFKFB4 functions mainly as a kinase and that, given the co-expression of PFKFB3 and PFKFB4, targeting both PFKFB3 and PFKFB4 isoforms using PFK158 and 5MPN may be a useful treatment option in cancer. 


\section{TABLE OF CONTENTS}

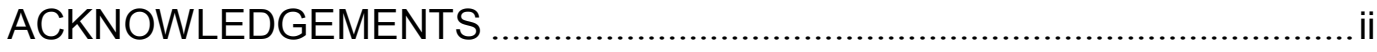

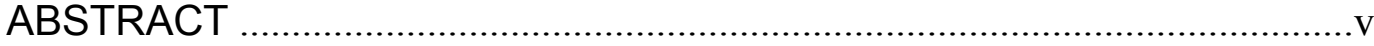

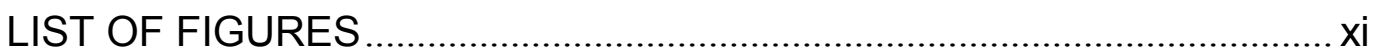

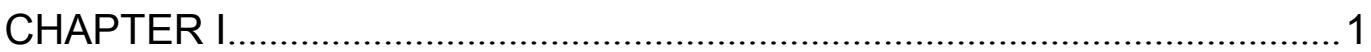

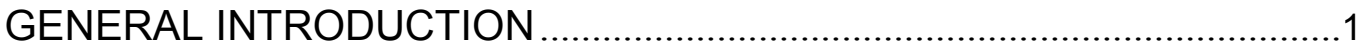

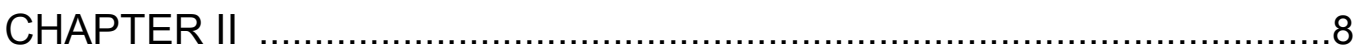

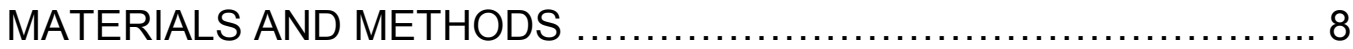

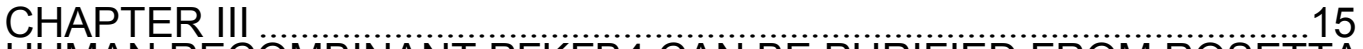

HUMAN RECOMBINANT PFKFB4 CAN BE PURIFIED FROM ROSETTAI COMPETENT ECOLI CELLS USING AFFINITY CHROMATOGRAPHY AND GEL FILTRATION

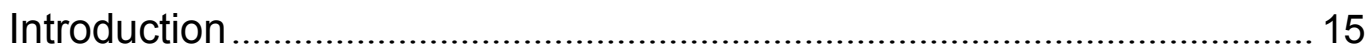

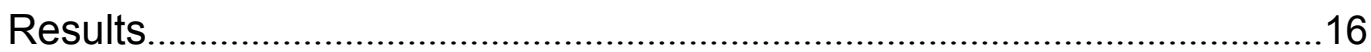

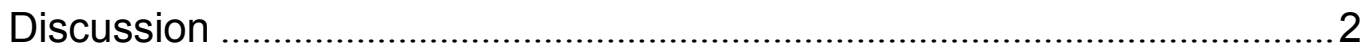

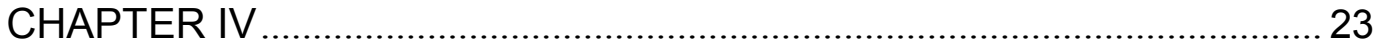

INTRODUCTION TO ENZYME KINETICS AND ENZYME INHIBITION

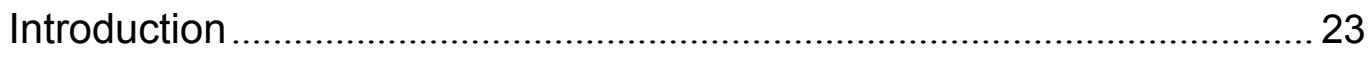

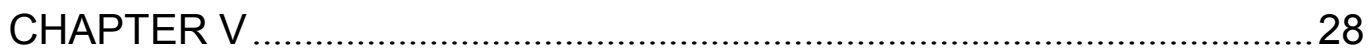

BOTH PFKFB4 AND PFKFB3 HAVE A HIGHER KINASE THAN BISPHOSPHATASE ACTIVITY, BUT THE KINASE ACTIVITY OF PFKFB4 IS LOWER

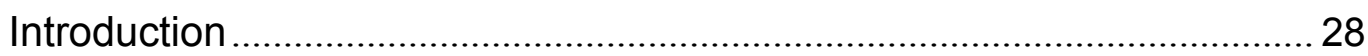

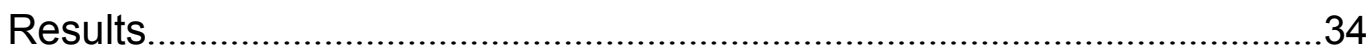

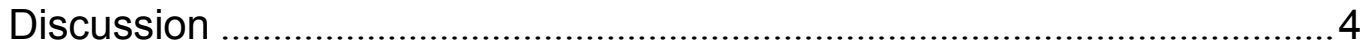

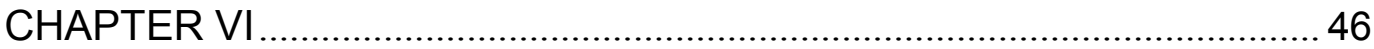

PFKFB4 IS INHIBITED BY THE SMALL MOLECULE INHIBITOR 5MPN AND NOT INHIBITED BY COMPOUNDS TARGETED AGAINST PFKFB3

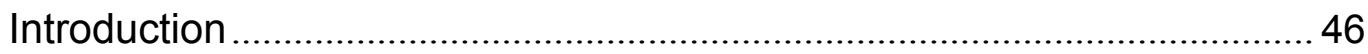

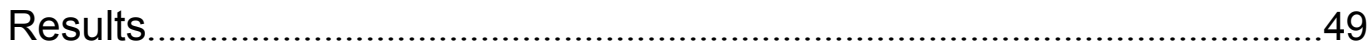

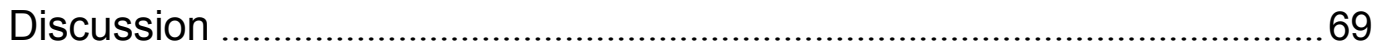

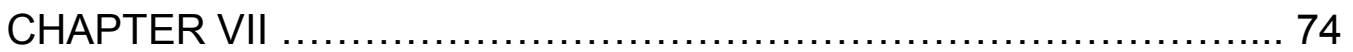

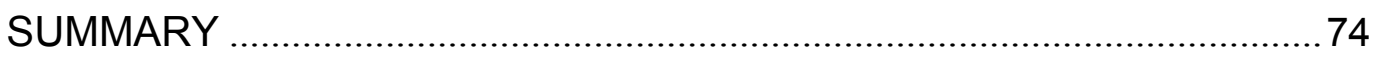

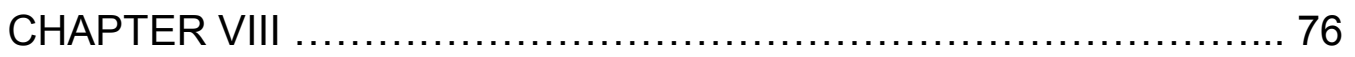

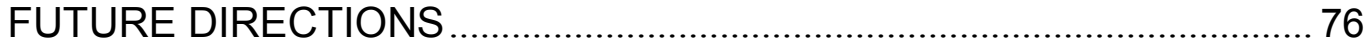

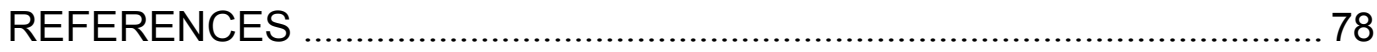

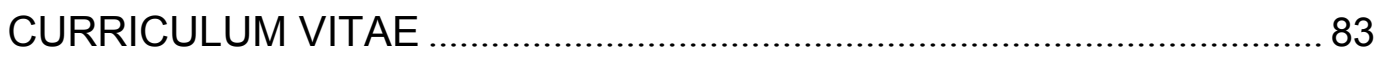




\section{LIST OF FIGURES}

Figure 1: Flow diagram of the fructose-2,6-bisphosphate assay

Figure 2: Designing primers to incorporate PFKFB DNA into expression vector

Figure 3: pET-24d expression vector construct designed to include kanamycin resistance, PFKFB4 DNA insert, and a His tag 19

Figure 4: Chromatogram showing the gel filtration fractions that yielded PFKFB4 protein

Figure 5: Coomassie stain of PFKFB4 protein ran on an SDS PAGE gel

Figure 6: Folding of PFKFB3 protein 30

Figure 7: Ribbon diagram of rat PFKFB4 32

Figure 8: The kinetic data of PFKFB4 fits the Michaelis-Menten curve with an $\mathrm{R}^{2}=0.99$ 36

Figure 9: The kinetic data of PFKFB3 fits the Michaelis-Menten curve with an $\mathrm{R}^{2}=0.99$ 38

Figure 10: The bisphosphatase domain of PFKFB4 has a maximum velocity of $11.7 \pm 0.82$ pmoles/minute

Figure 11: The bisphosphatase domain of PFKFB3 has a maximum velocity of $4.34 \pm 0.059$ pmoles/minute.

Figure 12: PFK15 competitively inhibits PFKFB3 and the $\mathrm{KI}$ is $2.1 \pm$ 0.5 .

Figure 13: The Lineweaver-Burk plot also suggests that PFK15 competitively inhibits PFKFB3. 
Figure 14: PFK15 competitively inhibits PFKFB4, but has a lower $\mathrm{K}_{\mathrm{i}}(10.8$ \pm 5.1 ) than that of PFKFB3

Figure 15: The Lineweaver-Burk plot also suggests that PFK15 competitively inhibits PFKFB4

Figure 16: PFK158 competitively inhibits PFKFB3 and the $\mathrm{KI}$ is $10.1 \pm 6.0$

Figure 17: The Lineweaver-Burk plot also suggests that PFK158 competitively inhibits PFKFB3.

Figure 18: PFK158 competitively inhibits PFKFB4, however, with a $\mathrm{KI}$ value of $34.2 \pm 14.6$, PFK138 better inhibits PFKFB3. 59

Figure 19: It is also confirmed through a Lineweaver-Burke plot that PFK158 blocks PFKFB4 through competitive inhibition

Figure 20: 5-MPN competitively inhibits PFKFB4, and with a KI value of $8.6 \pm 1.9$, is the best PFKFB4 inhibitor 62

Figure 21: The Lineweaver-Burk plot also suggests competitive inhibition for the interaction between PFKFB4 and 5-MPN 63

Figure 22: 5MPN does not inhibit PFKFB3 64

Figure 23: The addition of 5-MPN to the bisphosphatase assay for PFKFB4 increased the $\mathrm{V}_{\max }$ and $\mathrm{K}_{\text {cat }}$ 66

Figure 24: The addition of PFK15 to the bisphosphatase assay for PFKFB3 increased the $V_{\max }$ and $\mathrm{K}_{\text {cat }}$ 68 


\section{CHAPTER I}

\section{GENERAL INTRODUCTION}

\section{Cancer cells have an altered glucose metabolism}

The reprogramming of energy metabolism is an established hallmark of cancer cells [1]. Neoplastic cells exhibit an increased rate of glycolysis relative to normal cells, even in the presence of oxygen (i.e. aerobic glycolysis) [2]. This phenomenon of altered glucose metabolism in

cancer cells was first observed in the early $20^{\text {th }}$ century by Otto Warburg and was later termed the Warburg Effect. There are a number of theories addressing the question as to why tumors cells have higher glucose uptake and utilization.

Warburg theorized that the inability of an increased level of oxygen to inhibit the metabolism of glucose to lactate indicated that cancer cells had a defect in their mitochondria [3]. Although a few cancers have shown a genetic defect in key Krebs cycle enzymes, the evidence supporting Warburg's theory regarding the glycolytic phenotype in cancers is very limited [4]. Whereas Warburg's hypothesis involving mitochondrial defects as the cause of enhanced glycolysis in cancer cells may have been incorrect, mitochondrial dysfunction has been demonstrated to play a role 
in cancer [4] and there is growing interest in targeting mitochondrial function in order to inhibit tumor growth.

Another possible explanation for this phenomenon is that aerobic glycolysis provides the cancer cell with other metabolic requirements necessary for a thriving cancer cell [5]. For a cancer cell to grow and divide rapidly it requires an abundance of nucleotides, amino acids, and lipids. By increasing their glycolytic rate, cancer cells produce large amounts of the building blocks, such as glucose 6-phosphate, dihydroxyacetone phosphate, 3-phosphoglycerate, phosphoenolpyruvate and pyruvate that are used in the synthesis of these macromolecules [6].

It is clear that cancer cells alter their metabolism to a less efficient process in terms of energy production and that this switch offers an advantage to the cancer cell. This glycolytic phenotype has been confirmed in human studies through the use of positron emission topography in that cancer cells have higher glucose uptake than its surrounding normal cells. Additionally, it is clear that several different mechanisms exist that account for this increase in glycolytic flux, including the 6-phosphofructo-2-kinase/fructose-2,6-bisphosphatase family of enzymes [7]. 
The 6-phosphofructo-2-kinase/fructose-2,6- bisphosphatase family of enzymes is responsible for the cellular concentration of an activator of glycolysis

The enzyme phosphofructokinase-1 (PFK-1) is a key regulatory point in the glycolytic pathway. PFK-1 catalyzes the irreversible reaction of fructose-6-phosphate to fructose-1,6-bisphosphate and is able to respond to the energy needs of the cells [8]. For example, citrate and ATP inhibit PFK-1, while AMP activates its activity. The most potent allosteric activator of PFK-1 is fructose-2,6-bisphosphate (F-2,6-BP) which is able to overcome the inhibition caused by a high ATP concentration. F-2,6-BP levels are dictated by the 6-phosphofructo-2-kinase/fructose-2,6bisphosphatase (PFKFB1-4) family of enzymes that catalyze the interconversion of fructose-6-phosphate (F6P) and F-2,6-BP. There are four family members that share the same overall enzyme structure, but differ in their kinase and bisphosphatase activities due to structural differences in the regulatory regions [9].

In addition to their kinetic properties, each member of the PFKFB family is characterized by its tissue-specific expression. PFKFB1 and PFKFB2 isoforms are reported to have similar kinase to bisphosphatase ratios of $1.8: 1$ and $1.2: 2$ and were originally described to be expressed in the cells of the liver/muscle and kidney/pancreas respectively $[10,11]$. There is less characterization of PFKFB1 that links to cancer, however it has been shown to be induced in proliferating cells [12]. Furthermore, this induction results in an increased glycolytic rate [12]. PFKFB2 is known to 
be essential for the regulation of glycolysis in the heart and is regulated by adrenaline, insulin, anoxia and workload. Recent data suggests this isoform is co-expressed with other isoforms in normal body tissues and in tumors [9].

The most extensively studied family member is PFKFB3. PFKFB3 is of particular interest due to its high kinase to bisphosphatase ratio of 740:1 [13] and increased mRNA and protein expression in tumors relative to normal tissues [14]. Hypoxia inducible factor-1 (HIF-1) is commonly stabilized in cancer cells and causes an increase in transcriptional activity of the PFKFB3 gene [15]. Furthermore, phosphorylation enhances the kinase activity of PFKFB3 and is shown to be increased in a wide range of human cancer lines and correlates to increased cell proliferation in several cell lines, e.g. the COS7 kidney cell line [16]. This evidence, along with many other studies, has led to a surge of research into the characterization of PFKFB3 and examination of its potential as a cancer drug target.

Similar to PFKFB1 and PFKFB2, PFKFB4 has been less extensively studied than PFKFB3. However, more recently several studies examined its enzymatic activity and role in cancer development. In 2005, Minchenko et al. demonstrated that PFKFB4 mRNA is overexpressed in breast and colon malignant tumors compared to matched normal tissues and that the expression of the gene is highly induced by HIF-1 [17]. In 2010, in a nonbiased RNA interference screen to identify genes important to the survival of brain cancer stem-like cells 
(CSCs), PFKFB4 was identified as vital to the maintenance of brain CSCs and, thus a promising target in glioblastoma treatment [18]. A second nonbiased study further highlighted the role of PFKFB4 on cancer survival. Using siRNA-mediated gene silencing of several hundred metabolic proteins in three metastatic prostate cancer cell lines, Ros et al. identified PFKFB4 as a requirement for prostate cancer cell survival [19]. These three studies have drawn attention to the importance of PFKFB4 to cancer growth and led to growing interest in the development of inhibitors of PFKFB4 as possible anti-cancer agents.

\section{The kinase to bisphosphatase ratio of PFKFB4 is under investigation}

Although the requirement of PFKFB4 for the survival of a wide range of cancer types has been demonstrated by several studies, the exact function of PFKFB4 in the cell has not been fully established. Being a bifunctional enzyme, the balance between its kinase and bisphosphatase activities dictates its contribution to the concentration of F2,6-BP in a cell and thus to the regulation of glycolysis. If PFKFB4 has a higher bisphosphatase activity than kinase activity, then its expression would cause the concentration of F-2,6-BP in a cell to decrease, negatively impacting the allosteric activation of PFK-1, and increasing flux through the oxidative pentose phosphate pathway. Conversely, if the kinase domain of PFKFB4 were higher, the increased F-2,6-BP caused by its expression would result in the allosteric activation of PFK-1 and increased glycolytic flux. Evidence from Ros et al. demonstrate that 
PFKFB4 appears to function mainly as a bisphosphatase in prostate cancer cell lines. Conversely, Goidts et al. have demonstrated that PFKFB4 has more kinase activity in glioblastomas $[18,19]$. In addition, the direct examination of the relative kinase and bisphosphatase activities of PFKFB4 has, until now, been limited to experiments using enzyme purified from the rat testes. Our objective was to determine the relative kinase and bisphosphatase activities of human PFKFB4 and to then examine the effects of inhibition by small molecule inhibitors on PFKFB4 in comparison with effects on the PFKFB3 isoform. The ultimate goal of our studies is to investigate the PFKFB4 kinase domain as a potential target for the development of cancer therapies.

\section{PFKFB4 is required for the glycolytic phenotype and growth in tumor cells}

A recent examination of PFKFB4 activity presented strong evidence that supports the hypothesis that the predominant activity of PFKFB4 in cancer cells is to synthesize F-2,6-BP [20]. The selective inhibition of PFKFB4 in six of seven examined cancer cell lines led to decreased F2,6-BP synthesis, glycolysis, and ATP production. In addition, overexpression of PFKFB4 in each of these six cancer cell lines showed the converse effect of increasing production of F-2,6-BP, glycolysis, and ATP. These studies also showed that under hypoxic conditions, PFKFB4 was markedly induced (to a far greater extent than PFKFB3 protein levels) and siRNA-mediated knockdown PFKFB4 led to a significant increase in 
apoptosis indicating that PFKFB4 may be required for the glycolytic response to hypoxia. PFKFB4 expression also strongly correlated with expression of the hypoxia marker, carbonic anhydrase IX, by immunohistochemistry in xenograft tumors. In contrast, there was no observed correlation between PFKFB3 expression and the expression of carbonic anhydrase IX. Although the differences between the functions of PFKFB3 and PFKFB4 have yet to be fully examined, it appears that PFKFB4 may perform a unique function in cancer cells that are especially affected by hypoxia. [20] 


\section{CHAPTER II}

\section{MATERIALS AND METHODS}

\section{Vector Design}

The TOPO-PFKFB4 plasmid was extracted from DH5- a cells. Primers were designed to incorporate restriction sites at the ends of PCR amplified PFKFB4 gene. The PCR product was subject to a restriction digest and ligation in order to complete the pET-24d-PFKFB4 construct and was then confirmed using DNA sequencing. The new construct was transformed into Rosetta-I cells in order to express protein.

\section{Protein Expression and Purification}

The transformation was plated on an Luria-Bertani (LB) agar plate (kanamycin $50 \mu \mathrm{g} / \mathrm{ml}$ ). A colony was picked from the plate and grown in 10 $\mathrm{ml}$ of LB media (kanamycin $50 \mu \mathrm{g} / \mathrm{ml}$ ) at $37 \mathrm{C}$ overnight (300 RPM). The $10 \mathrm{ml}$ overnight culture was used to inoculate 21 LB cultures (kanamycin $50 \mu \mathrm{g} / \mathrm{ml}$ ). The cultures were placed in a $37 \mathrm{C}$ incubator (300 RPM) and the optical densities (OD) were checked hourly for absorbance at $600 \mathrm{~nm}$. Once the OD reached 1 (approximately 6 hours), protein 
expression was induced with $0.2 \mathrm{mM}$ of $\beta$-D-1-thiogalactopyranoside (IPTG). The $1 \mathrm{~L}$ cultures were grown overnight at $14 \mathrm{C}$ (300 RPM). The bacterial cultures were sonicated, then centrifuged at 25,000 RPM for 30 minutes at $4 \mathrm{C}$. Then, protein was extracted from the bacterial pellet using a lysis buffer (50 mM Tris buffer pH 7.5, $200 \mathrm{mM} \mathrm{NaCL}, 20 \mathrm{mM}$ imidazole, $0.35 \mathrm{mM} \beta$-mercaptoethanol). The protein was added to nickel agarose beads, washed 3 times with a bash buffer $(50 \mathrm{mM}$ Tris, $300 \mathrm{mM} \mathrm{NaCl}, 1$ $\mathrm{mM}$ imidazole, $0.175 \mathrm{mM} \beta$-mercaptoethanol). The protein was eluted with an elution buffer ( $50 \mathrm{mM}$ Tris, $300 \mathrm{mM} \mathrm{NaCl}, 200 \mathrm{mM}$ imidazole, $0.175 \mathrm{mM} \beta$-mercaptoethanol), and further purified through gel filtration.

\section{BCA Assay}

Bicinchoninic acid assay (BCA) reagents were prepared (Thermofisher, Rockford, IL) by combining 50 ul of reagent A with 1 ul of reagent B. Fifty $\mu \mathrm{l}$ per well was then added to a 96 well flat-bottom, clear plate (Falcon, San Jose, CA). Four $\mu$ l of sample was then transferred to the wells and the plate was incubated at $37^{\circ} \mathrm{C}$ for 30 minutes after which absorbance was read at $560 \mathrm{~nm}$ and a standard curve was used to interpolate the concentration of the protein sample.

\section{SDS PAGE Electrophoresis and Coomassie Stain}

Equal volumes of eluent was added to loading buffer (BioRad, Hercules, CA) containing $10 \mu \mathrm{l} / \mathrm{ml} \beta$-mercaptoethanol and then heated at $98{ }^{\circ} \mathrm{C}$ for 5 minutes and then loaded onto a 4-12\% gradient SDS-polyacrylamide gel 
(BioRad, Hercules, CA) and run for 60 minutes at 120 volts. The gel was then stained with Coomassie Brilliant Blue (BioRad, Hercules, CA) until the gel was a uniform blue color. The gel was then destained overnight in a destaining solution ( $5 \%$ methanol, $7.5 \%$ acetic acid, $\mathrm{dH}_{2} \mathrm{O}$ ).

\section{PFKFB3 protein}

Purified recombinant human PFKFB3 was obtained from the lab of Dr. Lee Hong-Hwan [28]. In brief, the His-tagged PFKFB3 protein was expressed in Escherichia coli BL21(DE3) pLysS. The protein was purified using nickel-affinity columns and Mono $Q$ anion-exchange chromatography [28].

\section{PFKFB4 protein}

PFKFB4 protein was purified according to the method outlined in chapter 1 of this thesis.

\section{Kinase Reaction}

Fructose-6-phosphate kinase activities of human recombinant PFKFB3 and PFKFB4 were assayed using previously described methods wherein one unit of activity was defined as the amount of enzyme that catalyzes the formation of $1 \mu \mathrm{mol}$ of F-2,6-BP per min [31]. $500 \mathrm{ng}$ of PFKFB3 was assayed, while $5 \mu \mathrm{g}$ of PFKFB4 was used for the reaction. Aliquots of the reaction mixture were removed at intervals, added to $0.1 \mathrm{~N} \mathrm{NaOH}$ and then neutralized to a pH of 7.2 and assayed for F-2,6-BP as described below. 


\section{Fructose-2,6-Bisphosphate Assay}

F-2,6-BP content was measured using a coupled enzyme reaction following the method of Van Schaftingen et al. [33]. Briefly, aliquots from the kinase assay were neutralized to a $\mathrm{pH}$ of 7.2 using $1 \mathrm{M}$ acetic acid and $1 \mathrm{M}$ Hepes. Then, they were incubated at $25{ }^{\circ} \mathrm{C}$ for $2 \mathrm{~min}$ in $50 \mathrm{mM}$ Tris, 2mM Mg2+, 1mM F6P, 0.15mM NAD, 10u/l PPi-dependent PFK1, 0.45 $\mathrm{kU} / \mathrm{l}$ aldolase, $5 \mathrm{kU} / \mathrm{l}$ triosephosphate isomerase, and $1.7 \mathrm{kl} / \mathrm{l}$ glycerol-3phosphate dehydrogenase. $0.5 \mathrm{mM}$ pyrophosphate was added and the rate of change in absorbance $(O D=339 \mathrm{~nm})$ per min over five minutes was determined (see diagram below). A calibration curve using 0.1 to $1 \mathrm{pmol}$ of F-2,6-BP (Sigma, St. Louis,MO) was used to calculate total F-2,6-BP present in each aliquot. 
Figure 1. Flow diagram of the fructose-2,6-bisphosphate assay. The reaction mix contains the sample to be quantified for F-2,6-BP concentration, 50mM Tris, $2 \mathrm{mM}$ Mg2+, 1mM F6P, 0.15mM NAD, 10u/l PPi-dependent PFK1, $0.45 \mathrm{kU} / \mathrm{l}$ aldolase, $5 \mathrm{kU} / \mathrm{l}$ triosephosphate isomerase, and $1.7 \mathrm{kl} / \mathrm{l}$ glycerol-3-phosphate dehydrogenase, and $0.5 \mathrm{mM}$ pyrophosphate. The rate of change of absorbance $(O D=339 \mathrm{~nm})$ per min over two minutes was determined.

\section{Figure 1}
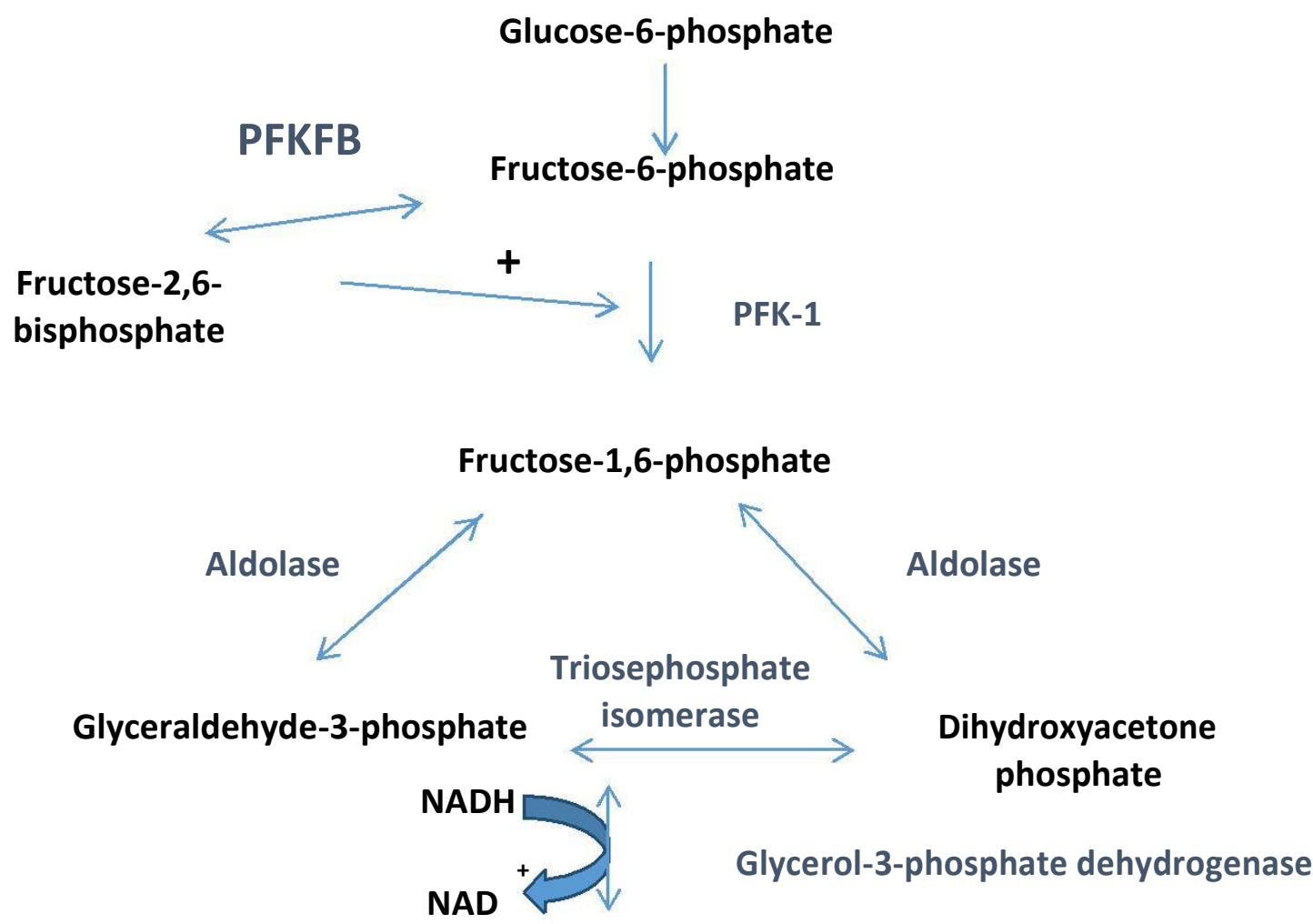

Glycerol-3-phosphate 


\section{Bisphosphatase Reaction}

The bisphosphatase activities of human recombinant PFKFB3 and PFKFB4 were assayed using previously described methods wherein one unit of activity was defined as the amount of enzyme that catalyzes the formation of $1 \mu \mathrm{mol}$ of F-6-P per min [33]. $10 \mu \mathrm{g}$ of PFKFB3 and PFKFB4 were assayed in the reaction. Aliquots of the reaction mixture were removed at intervals, added to $0.1 \mathrm{~N} \mathrm{NaOH}$ and then neutralized to a $\mathrm{pH}$ of 7.2 and assayed for F-6-P using the method of Van Schaftingen et al. [33].

\section{Drug Treatment}

\section{PFK15}

PFK15 (1-(4-pyridinyl)-3-(2-quinolinyl)-2-propen-1-one) is a PFKFB3 small molecule inhibitor derivative of 3PO (3-(3-pyridinyl)-1-(4-pyridinyl)-2propen-1-one) and was obtained from the lab of Dr. Jason Chesney [41]. For the kinase assay, three concentrations of PFK15 were used, $0.1 \mu \mathrm{M}, 1$ $\mu \mathrm{M}$, and $10 \mu \mathrm{M}$. For the phosphatase assay of PFKFB3, $10 \mu \mathrm{M}$ of PFK15 was used.

\section{$\underline{\text { PFK158 }}$}

PFK158 is a second small molecule inhibitor of PFKFB3 derived from PFK15 that we obtained from the lab of Dr. Jason Chesney [43]. For the 
kinase assay, three concentrations of PFK158 were used, $0.1 \mu \mathrm{M}, 1 \mu \mathrm{M}$, and $10 \mu \mathrm{M}$.

\section{5-MPN}

5MPN is a novel and unpublished inhibitor of PFKFB4 that was identified through the combination of computational modeling and receptor based in silico screening. For the kinase assay, three concentrations of 5MPN were used, $0.1 \mu \mathrm{M}, 1 \mu \mathrm{M}$, and $10 \mu \mathrm{M}$. For the phosphatase assay of PFKFB4, $10 \mu \mathrm{M}$ of $5 \mathrm{MPN}$ was used. 


\section{CHAPTER III}

\section{PURIFCATION OF HUMAN PFKFB4 FROM E. COLI CELLS USING AFFINITY CHROMATOGRAPHY AND GEL FILTRATION}

\section{INTRODUCTION}

The four members of the PFKFB family were originally believed to be expressed in distinct tissues. The enzymes encoded by the PFKFB1, PFKFB2 and PFKFB4 genes were originally described by the tissue in which they were thought to be exclusively expressed; liver/muscle, heart, and testes respectively. Additionally, these three isoenzymes have been reported to have very similar kinase to bisphosphatase ratios [21]. The kinase to bisphosphatase ratio of the final member, PFKFB3, originally termed the placental PFK2 or ubiquitous PFK2 [22] is reported to be approximately $740: 1$ [13], substantially higher than that of the other three PFKFB family members.

While the core structure of the PFKFB enzymes is highly conserved, the observed differences between the enzymatic activities of the different isozymes arise from structural variations in the terminal regions that are believed to cause regulatory effects on the catalytic 
regions by altering their tertiary and quaternary conformations [22]. Since co-expression of several isoforms has been noted in multiple tissues, it has been suggested that each family member may have unique roles under different physiological conditions or have differing responses to specific stimuli [15].

Understanding the precise kinase and bisphosphatase activities of PFKFB4 is crucial for determining whether it may be a rational drug target for cancer therapy. Analysis of PFKFB4 enzymatic activity has thus far been limited to protein purified from rat testes. In 1991, Sakata et al. examined the enzymatic activity of PFKFB4 using protein purified from the rat testes and determined that its kinase domain has 4.1 times greater activity than its bisphosphatase domain [23]. Although there exists 97 percent homology between the rat and human PFKFB4 sequence [24], human recombinant PFKFB4 protein has yet to be examined. In order to adequately examine the enzymatic activity of human PFKFB4, we first sought to prepare human recombinant PFKFB4. In this chapter, we describe our experiments that were used in the preparation and purification of human recombinant PFKFB4.

RESULTS

The human PFKFB4 protein was expressed in Rosetta-1 cells and purified using nickel-agarose beads and gel filtration 
The TOPO-PFKFB4 plasmid was extracted from DH5a cells. To construct a plasmid containing the PFKFB4 gene we first designed primers in order to incorporate restriction sites into the DNA for the purpose of cloning into our expression vector. The sense primer (Figure 2a) was designed to incorporate a Nco1 restriction site into the 3 ' end of the DNA insert. Additionally, an antisense primer (Figure 2b) was designed to incorporate a Xho1 restriction site into the 5' end PFKFB4

DNA insert. The PCR product was subject to a restriction digest and ligation in order to complete the pET-24d-PFKFB4 construct and was confirmed using DNA sequencing. These two restriction sites were chosen so that the PFKFB4 DNA insert could be inserted into the pET-24d expression vector with a His-tag at the c-terminus (Figure 3). Rosetta-I competent cells were transformed with the expression vector and then conditions for the optimal expression of the PFKFB4 protein were tested. Protein expression was induced with $0.5 \mathrm{mM}$ Isopropyl $\beta-\mathrm{D}-1$ thiogalactopyranoside (IPTG) and then purified through affinity chromatography using a nickel-agarose column.

The protein was further purified through gel filtration which separated our protein of interest from a similarly sized heat-shock protein (Figure 4). Gel filtration fractions containing the PFKFB4 protein were subjected to SDS-PAGE electrophoresis to check for protein expression and purity (Figure 5). The protein concentration was determined using the BCA biochemical assay. 
Figure 2: Designing primers to incorporate PFKFB4 DNA into expression vector: a. Sense primer designed to incorporate a Ncol restriction site into the PFKFB4 DNA insert, b. Antisense primer designed to incorporate Xhol restriction site into the PFKFB4 DNA insert

\section{Figure 2}

a.

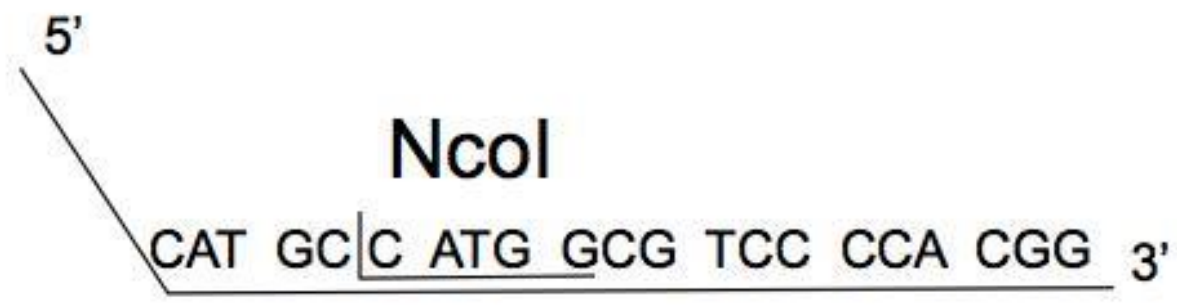

b.

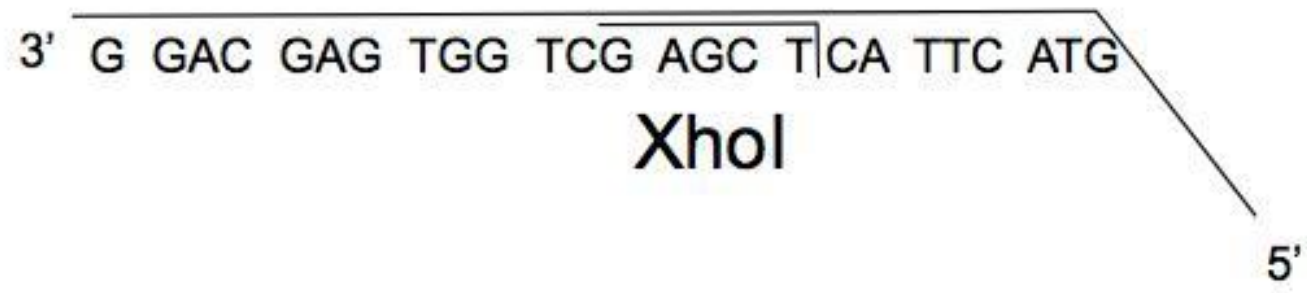


Figure 3: pET-24d expression vector construct designed to include PFKFB4 DNA insert and a His-tag. The restriction sites Ncol and Xhol were chosen because they allowed the PFKFB4 insert to be incorporated into the plasmid with a His-tag at the $C$ terminus and a few amino acids before and after the inserted gene.

\section{Figure 3}

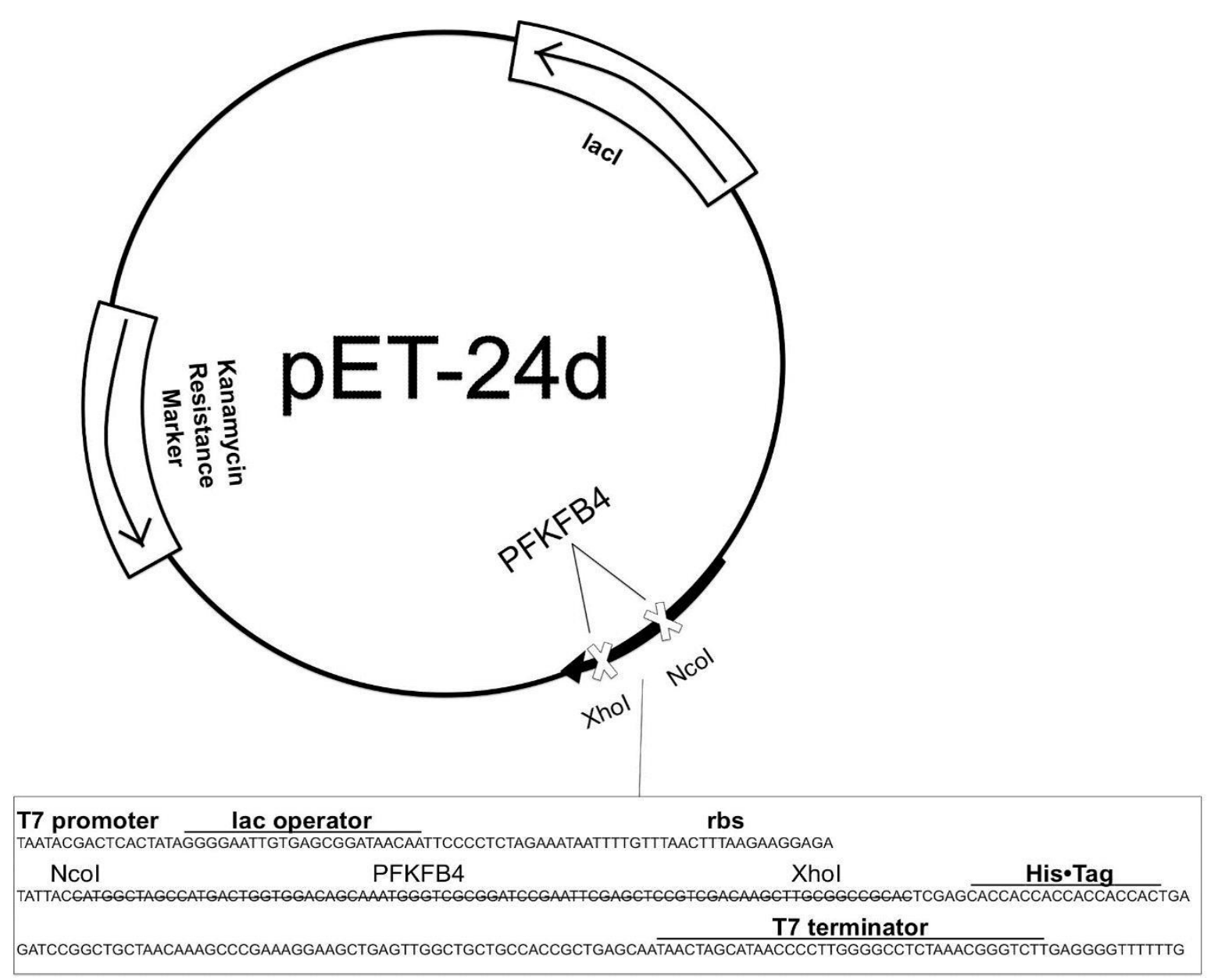


Figure 4. Chromatogram showing the gel filtration fractions that yielded PFKFB4. The protein was purified through gel filtration to separate it from the heat shock protein that is close in size to PFKFB4. Fractions 23-29 were the fractions determined to contain the PFKFB4 protein.

\section{Figure 4}

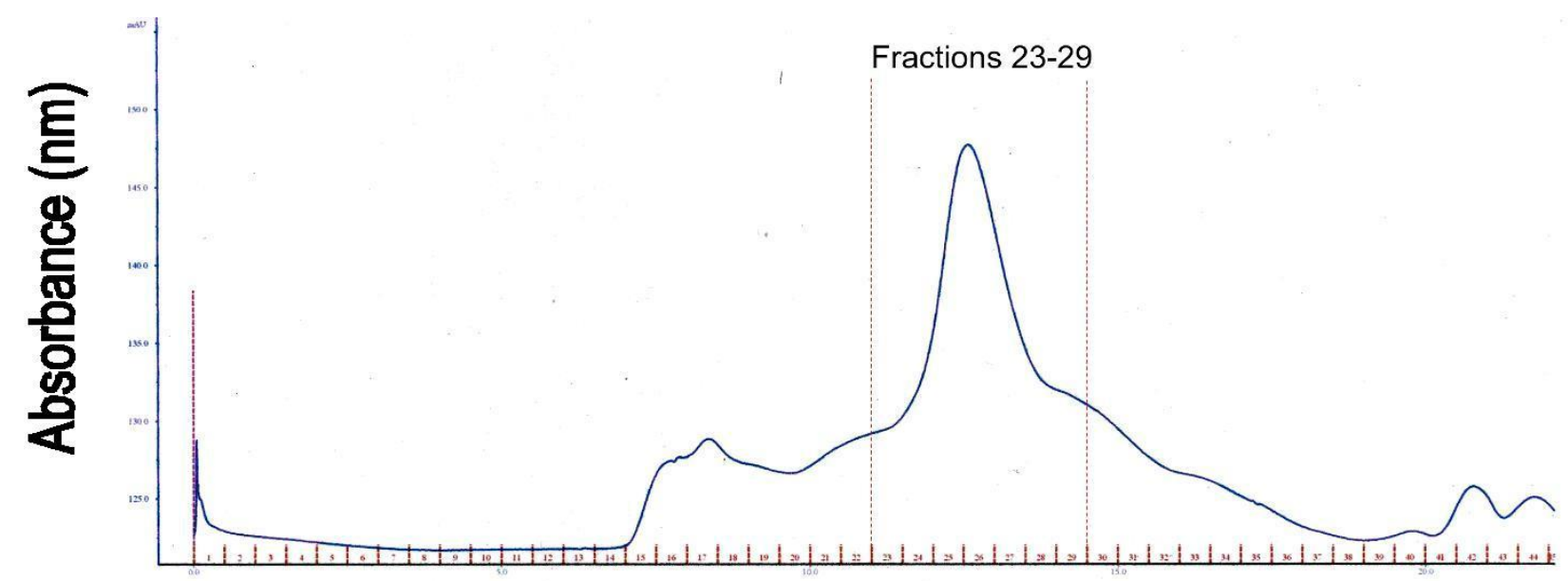

Fraction Number 
Figure 5. Coomassie stain of PFKFB4 protein on 4-20\% SDS PAGE gel. After the protein was purified through gel filtration, fractions 23-29 were assayed for purity using SDS PAGE and Coomassie blue staining.

\section{Figure 5}

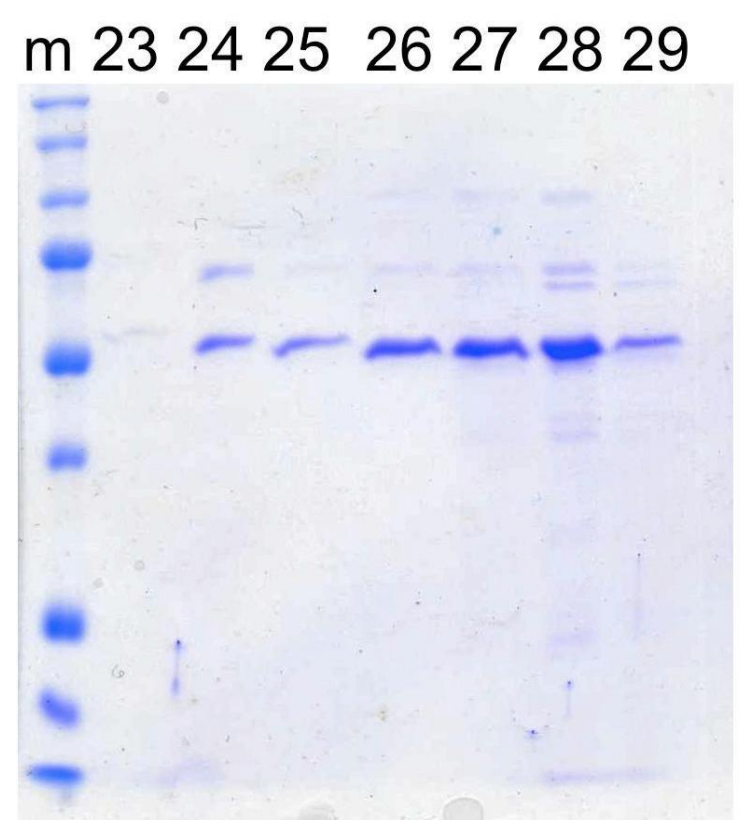




\section{DISCUSSION}

In order to adequately examine the function of human PFKFB4, we first set out to prepare recombinant human PFKFB4. After obtaining a human TOPO-PFKFB4 DNA construct, our first goal was to isolate the PFKFB4 gene using restriction digestion and then ligating the DNA into a pET-24d expression vector. This expression vector contains a c-terminal His-Tag sequence. The effect of the His-tag on the three dimensional folding and activity has not been explored, but its addition is crucial for purifying the PFKFB4 protein from a whole cell lysate using nickel-agarose column. After purifying the PFKFB4 protein using affinity chromatography, we further purified our product using gel filtration. The protein purity was assessed using SDS-PAGE electrophoresis and the gel was stained with Coomassie brilliant blue. A prominent protein band was visible at $\sim 55 \mathrm{kD}$ which is the reported size of the PFKFB4 protein, with no evidence of contaminating protein in any of the fractions. Thus, our experiments resulted in the purification of recombinant PFKFB4 protein for subsequent use in assays to test its enzymatic properties. 


\section{CHAPTER IV}

\section{INTRODUCTION TO ENZYME KINETICS AND ENZYME INHIBITION}

\section{Enzyme Kinetics}

Enzymes are proteins that catalyze chemical reactions. For singlestep reaction, according to the law of mass of action, the rate of the reaction is proportional the concentration of reactants. To characterize the relationship between substrate concentration (S) and reaction velocity (v), Lenor Michaelis and Maud Menten developed the following equation [44]:

$$
v=\frac{d[P]}{d t}=\frac{V_{\max }[S]}{K_{m}+[S]} .
$$

This relationship is known as the Michaelis-Menten equation and $V_{\max }$ represents the maximum reaction rate of the system at saturating substrate concentrations. Furthermore, the Michaelis-Menten constant, $\mathrm{K}_{\mathrm{m}}$, is the substrate concentration when the reaction rate is half the $V_{\max }$. Additionally, the value of $\mathrm{Km}$ is inversely related to the substrate's affinity for the enzyme. For instance, a high $\mathrm{Km}$ indicates a low affinity and that 
the rate will approach the $V_{\max }$ more slowly. The relationship between $V_{\max }$ and $\mathrm{Km}$ is clarified upon observation of the following graph [44]:

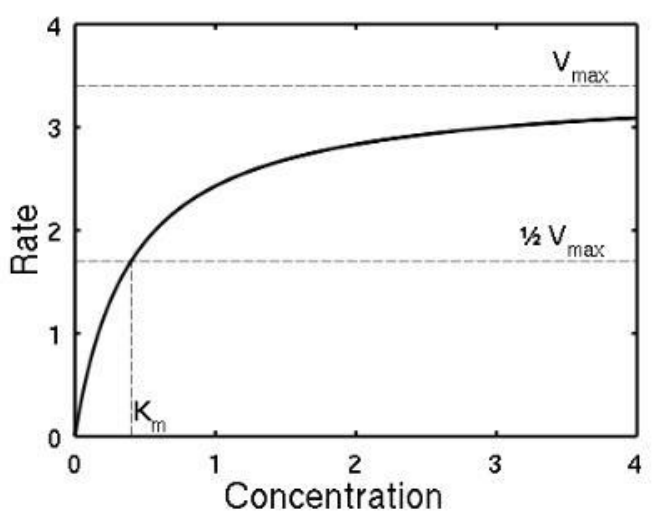

Reaction rates increase with increasing substrate concentrations, asymptotically approaching $V_{\max }$. Single-substrate reactions are also characterized by their turnover number (Kcat), which is described as the maximum number of substrate molecules converted to product per enzyme molecule per second. The relationship between $\mathrm{K}_{\mathrm{cat}}$, enzyme concentration (ET), and maximum velocity is described by the following equation [44]:

$$
\mathrm{k}_{\text {cat }}=\frac{\mathrm{V}_{\text {max }}}{[\mathrm{E}]_{\mathrm{T}}}
$$

The units of $\mathrm{K}_{\mathrm{cat}}$ is the rate of substrate converted to product per unit time per enzyme concentration. Typically, the units for substrate over enzyme concentration are dropped and only the units $\sec ^{-1}$ are used. One constant commonly used to describe enzymatic activity is the specificity constant, $\mathrm{K}_{\mathrm{cat}} / \mathrm{Km}$. This constant is a measurement of how efficiently an 
enzyme converts a substrate to product and is used to compare enzymes [45]. Theoretically, the specificity constant has an upper limit of $10^{8}-10^{10}$ $M$ and enzymes that approach this limit are considered more efficient.

The enzyme constants, $V_{\max }$ and $\mathrm{K}_{\mathrm{m}}$, are determined experimentally by running a series of enzymatic assays at varying substrate concentrations and then measuring reaction rates. The $\mathrm{V}_{\max }$ and $\mathrm{K}_{\mathrm{m}}$ are then calculated by plotting reaction rate versus substrate concentration. Then, using nonlinear regression of the Michaelis-Menten equation, the enzyme constants can be calculated. However, before the use of computers, calculating enzyme constants involved graphical methods. One of these methods involves the linearization of the Michaelis-Menten equation through a double reciprocal plot or Lineweaver-Burk plot. This plot can be visualized through the following graphical representation [44]:

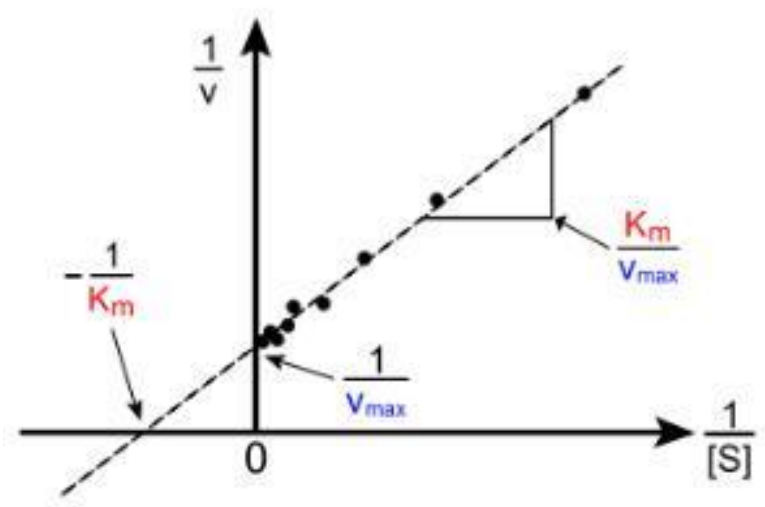

From this plot, one can calculate the $\mathrm{Km}_{\mathrm{m}}$ and $\mathrm{V}_{\max }$ constants through points where the line pass the axes. For instance, the $x$-intercept is equal to $-1 / \mathrm{K}_{\mathrm{m}}$ and the $\mathrm{y}$ intercept is $1 / \mathrm{V}_{\max }$. 


\section{Enzyme Inhibition}

Enzyme activity depends on a variety of factors, such as enzyme concentration, substrate concentration, $\mathrm{pH}$ of the reaction, temperature, and presence of activators or inhibitors [45]. Enzyme inhibitors are usually low molecular weight molecules that interact with the enzyme and change the activity of the enzyme by slowing down the reaction rate [45]. Inhibitors can bind to and inhibit a specific enzyme or can inhibit the enzyme nonspecifically through physical or chemical changes that denature the protein portion of the enzyme [46]. Based on how the enzyme interacts with the inhibitor, the inhibitor can be classified as either reversible if their interaction with the enzyme is non-covalent, or irreversible if the interaction is covalent. Furthermore, reversible inhibitors can be further classified as uncompetitive, competitive, or non-competitive.

Competitive inhibitors usually mimic the enzyme's natural substrate and compete with the substrate for access to the active site. Additionally, the inhibitor can only bind to the free enzyme and not the enzymesubstrate complex. Therefore, by increasing the substrate concentration in the reaction mixture, the substrate can out-compete the inhibitor for the binding site. In contrast to competitive inhibitors, uncompetitive inhibitors only bind to the enzyme-substrate complex. And unlike competitive inhibition which reduces only the $\mathrm{Km}$, in uncompetitive inhibition, both the $\mathrm{Km}$ and the $V_{\max }$ are reduced. In noncompetitive inhibition, the inhibitor 
binds to the enzyme at a site other than the active site and does not interfere with the binding of the substrate to the enzyme's active site.

Hence, the degree of inhibition is dependent only on the concentration of inhibitor added to the reaction and only the $V_{\max }$ is reduced. The last type of reversible inhibition is a mixed type of inhibition. In this case, an inhibitor can bind to both the free enzyme as in competitive inhibition and to the enzyme substrate complex as in uncompetitive inhibition. This type of inhibition interferes with the binding of the substrate to the enzyme and reduces the effectiveness of the enzyme turnover [46]. Therefore, the $\mathrm{K}_{\mathrm{m}}$ is increased, but the $V_{\max }$ is reduced.

To evaluate the potency of an inhibitor the inhibition constant, $\mathrm{K}_{\mathrm{i}}$, is calculated [46]. The $\mathrm{K}_{\mathrm{i}}$ is the concentration of enzyme required to produce half that maximum inhibition. Therefore, the lower the $\mathrm{K}_{\mathrm{i}}$ value, then the lower the concentration of inhibitor needed to lower the reaction rate. The precise formula used to calculate the Ki value depends on the mode of inhibition (competitive, noncompetitive, uncompetitive, or mixed inhibition). However, once the mode of inhibition is determined, the $\mathrm{K}_{\mathrm{i}}$ can be calculated and the strength of the inhibitor to an enzyme can be determined. 


\section{CHAPTER V}

\section{BOTH PFKFB4 AND PFKFB3 HAVE A HIGHER KINASE THAN BISPHOSPHATASE ACTIVITY, BUT THE KINASE ACTIVITY OF PFKFB4 IS LOWER}

\section{INTRODUCTION}

$\mathrm{F}-2,6-\mathrm{BP}$ is an allosteric activator of PFK-1 found in all mammalian tissues $[25,26]$. The concentration of this signaling molecule is determined by the kinase activity of the PFKFB family of enzymes. The synthesis of F-2,6-BP from F-6-P is catalyzed by the N-terminal domain of the enzyme and is a sequential order reaction that involves a transfer of a phosphate from ATP to F-6-P [27]. Site-directed mutagenesis and modeling has provided evidence that the kinase domain of PFKFB is structurally similar to the mononucleotide-binding protein family which contain members such as adenylate kinase (AK) and ras [9]. Unlike AK, the kinase domain of PFKFB family members functions as a homodimer in which the two central six stranded $\beta$-sheets interact to form a continuous inter-monomer $\beta$-sheet (see figure below) [9]. But similar to AK, it is 
proposed that ATP and F-6-P are trapped within the kinase domain and the mechanism is likely to include an induced fit feature [9].

Since PFKFB3 is the best characterized of the PFKFB family, in this study we decided to focus on comparing the enzymatic activity of PFKFB4 to that of PFKFB3. PFKFB3 has a significantly higher kinase to bisphosphatase ratio than PFKFB4 and there are several structural differences between the PFKFB3 and PFKFB4 that account for this difference. Structural and sequence variations between the two family members alter their catalytic and regulatory properties. PFKFB3 and PFKFB4 share a high sequence homology in their core kinase domain, but the surrounding $\mathrm{N}$-terminal regulatory domains have important differences that alter their overall kinase functions [7]. The PFKFB3 isoform contains a $\beta$-hairpin $\mathrm{N}$-terminal region that interacts with regions of the bisphosphatase domain affecting the function of the bisphosphatase catalytic domain (Figure 6) [28]. 
Figure 6: Folding of PFKFB3 protein. A ribbon diagram of the dimeric PFKFB3 structure is shown. ADP is bound to the kinase pocket (magenta) and F-6-P is shown bound to the kinase pocket. The regulatory domain is shown in green [28].

\section{Figure 6}

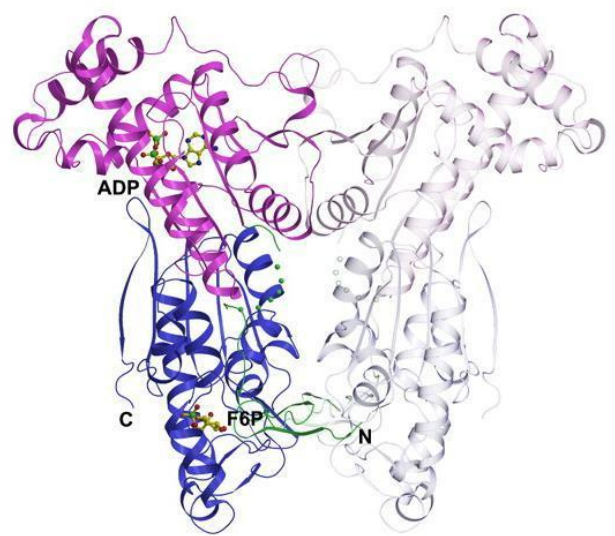


Additionally, sequence differences around the ATP and F-6-P binding sites decrease the flexibility of the C-terminus and its interference with the kinase catalytic region $[28,29]$. Because of these structural features, the PFKFB3 isoform has a high kinase activity compared to the other isoforms. The $V_{\max }$ of the kinase activity is reported as $142 \mathrm{mU} / \mathrm{mg}$, the $\mathrm{K}$ m as $32 \mu \mathrm{M}$ and kinase to bisphosphatase ratio as $710: 1$ for the human PFKFB3 isoform [13]. In contrast, the rat testes PFKFB4 isoform has been observed to have a kinase activity $V_{\max }$ of $90 \mathrm{mU} / \mathrm{mg}, \mathrm{Km} 85 \mu \mathrm{M}$ and kinase to bisphosphatase ratio of 4.1:1. Although the crystal structure of human PFKFB4 has yet to be solved, the structure of PFKFB4 has been reported for PFKFB4 from rat testis (figure 7). 
Figure 7: Ribbon diagram of rat PFKFB4. The protein monomers are depicted as red and green. The ATPyS and $\mathrm{PO}_{4}$ ligands are shown in black. Arrrangement of the functional dimer about the crystallographic twofold axis of rotation. The protein monomers are colored red and green, and the ATPyS and $\mathrm{PO}_{4}$ ligands are in black.

\section{Figure 7}

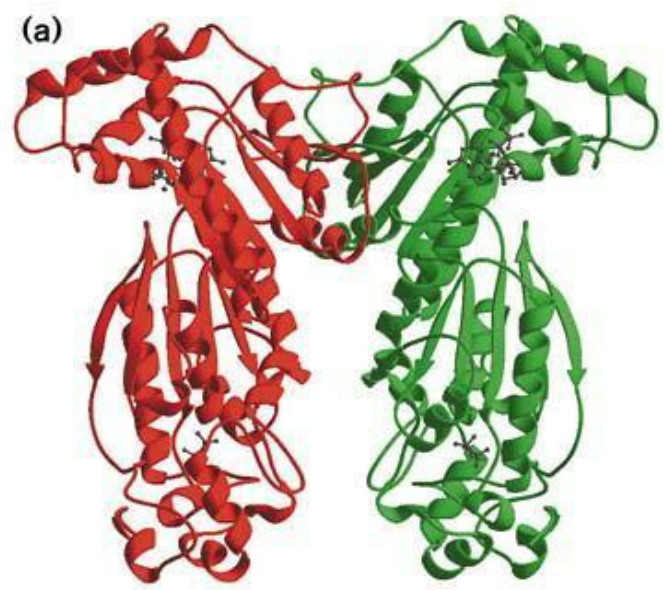


Sequence variations in PFKFB3 and PFKFB4 also contribute to regulatory differences between the two isoforms. In the PFKFB4 isoform, ATP binding must occur before F-6-P can bind making PFKFB4 more sensitive to ATP than F-6-P concentrations [30]. The sequence differences between PFKFB4 and the other isoforms affect how it is regulated. There is a mutation in the PFKFB4 isoform which destroys the recognition sequence for the cAMP-dependent protein kinase [31]. Additionally, unlike PFKFB3, PFKFB4 is not phosphorylated by any kinases in vivo [31]. PFKFB3 is phosphorylated by AMPK which activates its kinase activity [9].

Being bifunctional enzymes, all members of the PFKFB family have both a kinase and bisphosphatase activity. The bisphosphatase domain functions to catalyze the hydrolysis of F-2,6-BP to F-6-P and $\mathrm{Pi}$ and when the bisphosphatase activity is higher than the kinase activity, F-2,6-BP is converted to fructose-6-phosphate with a consequents of a decrease in the glycolytic flux. Additionally, enzymatic studies have shown that F-6-P is a non-competitive inhibitor of the reaction [36]. Furthermore, F-6-P is able to stabilize the intermediate by forming a complex that excludes $\mathrm{H}_{2} \mathrm{O}$ from the active site and preventing hydrolysis of the phosphoenzyme intermediate [37, 38].

Sequence differences between PFKFB3 and PFKFB4 also modify their bisphosphatase activities. The $V_{\max }$ of the bisphosphatase activity is reported as $0.2 \mathrm{mU} / \mathrm{mg}$, the $\mathrm{Km}$ for F-2,6-BP as $130 \mu \mathrm{M}$ [13]. Although the 
enzymatic activity of the human form of PFKFB4 has not been determined, the rat form of PFKFB4 is reported to have a $V_{\max }$ of the bisphosphatase activity is reported as $22 \mathrm{mU} / \mathrm{mg}$, the $\mathrm{Km}$ for F-2,6-BP is $21 \mu \mathrm{M}$ and kinase to bisphosphatase ratio as 4.1:1.

Using the human recombinant PFKFB3 and PFKFB4 in the same kinase and bisphosphatase assays will provide evidence as to which domain is more active in PFKFB4. The direct examination of the relative kinase and bisphosphatase activities of PFKFB4 has, until now, been limited to experiments using enzyme purified from the rat testes. My objective here is to determine the bisphosphatase activity of human PFKFB4. This data will then provide evidence as to the importance of PFKFB4 as a potential target for the development of cancer therapies.

\section{RESULTS}

\section{The kinase domain of recombinant human PFKFB4 has a maximum velocity of $25.2 \pm 0.93$ pmoles/minute}

We assayed the kinase and phosphatase activities of PFKFB4 using purified recombinant human PFKFB4. The kinase activity was examined by measuring the rate of production of F-2,6-BP by PFKFB4 from F6P as described by Van Schaftingen et al. using a range of substrate concentrations derived from the published rat PFKFB enzyme data (Figure 8). The $V \max$ was determined to be $25.2 \pm 0.93$ pmoles/minute and the $\mathrm{Km} 356 \pm 44.7 \mu \mathrm{M}$ (95\% confidence level). We 
next measured the catalytic constant (Kcat), which measures the number of substrate molecules turned over per enzyme molecule per second and is defined as the Vmax per concentration of enzyme. The Kcat for PFKFB4 was calculated to be $5.03 \pm 0.19 \mathrm{sec}^{-1}$. In order to define the catalytic efficiency of the PFKFB4 enzyme, we also calculated the specificity constant for PFKFB4 from the Km and Kcat values to be 0.0141 $\mu \mathrm{M} /$ second. 
Figure 8. The kinetic data of PFKFB4 fits the Michaelis-Menten curve with an $\mathbf{R}^{\mathbf{2}}=\mathbf{0 . 9 9}$. The velocity of the kinase reaction was determined using a range of F-6-P concentrations (10, 42.5, 85, 170, 340, 680, 2000, and $6000 \mu \mathrm{M})$ and the kinetic constants were calculated from these data (95\% confidence level).

\section{Figure 8}

$M$ ic $h$ a e lis $-M$ e $n$ te $n$ :

P F K F B $4 \mathrm{~K}$ in a s e A c tiv ity

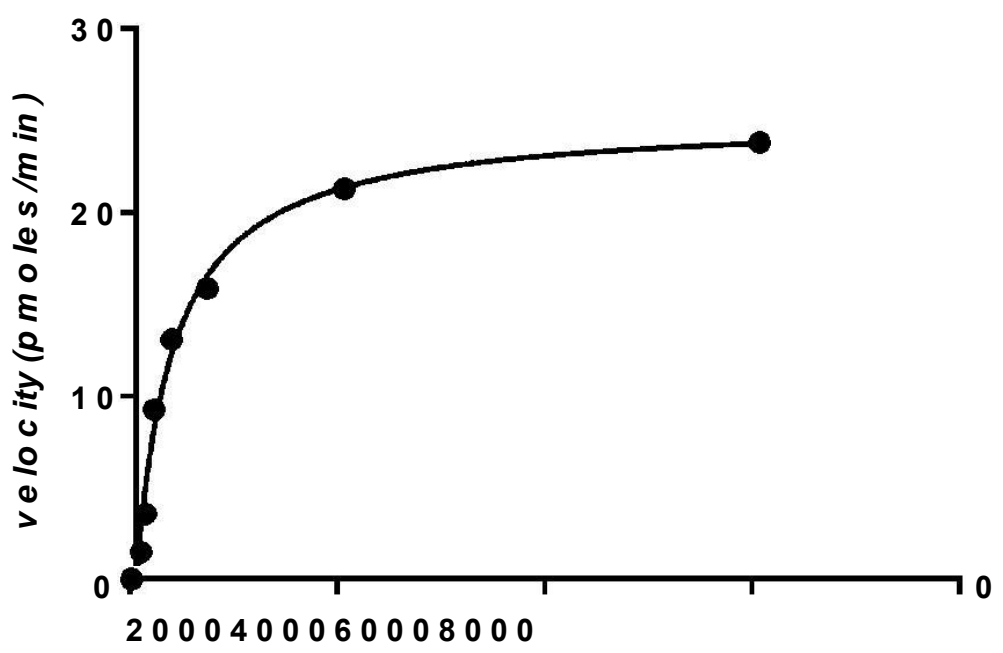

[F r u c to s e $-6-p h$ os $p h$ a te ] u M 


\section{The kinase domain of recombinant human PFKFB3 has a maximum}

velocity of $23.2 \pm 0.82$ pmoles/minute.

In order to compare the kinase domain of PFKFB4 to that of the PFKFB3 isoform which is known to have a far higher kinase activity, we next examined the kinase activity of recombinant human PFKFB3. We used $0.5 \mu \mathrm{g}$ of PFKFB3 protein and 6 concentrations of F-6-P to run the reactions and then assayed the reaction rate under each condition using an enzyme-coupled reaction that determined production formation for each condition (Figure 9). The Vmax was determined to be $23.2 \pm 0.82$ pmoles/minute and the $\mathrm{Km} 10.2 \pm 1.5 \mu \mathrm{M}$ (95\% confidence level). The

Kcat was calculated to be $46.5 \pm 1.63 \mathrm{sec}^{-1}$, and the specificity constant to be $4.55 \mu \mathrm{M} /$ second. 
Figure 9. The kinetic data of PFKFB3 fits the Michaelis-Menten curve with an $\mathbf{R}^{\mathbf{2}}=\mathbf{0 . 9 9}$. The velocity of the kinase reaction for PFKFB3 was determined under a range of F-6-P concentrations $(1,5,10,40$, and 80 $\mu \mathrm{M})$ and kinetic constants were calculated from these data.

\section{Figure 9}

$M$ ic $h$ a e lis - M e $n$ te $n$ :

$P F K F B 3 K$ in a s e A c tiv ity

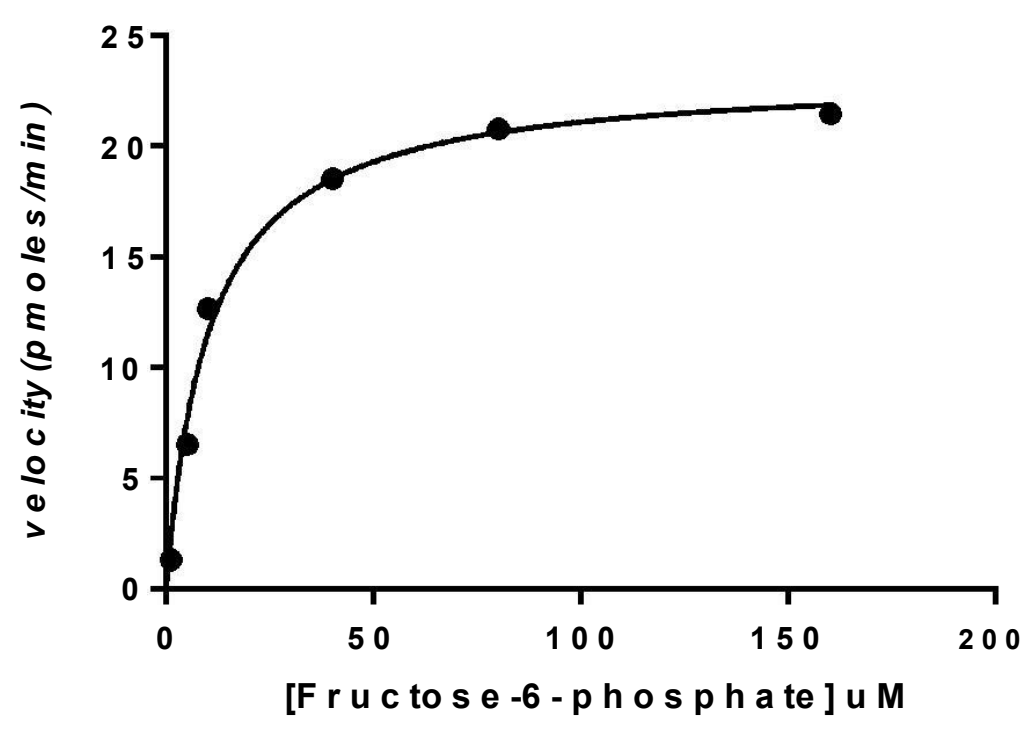




\section{The bisphosphatase domain of recombinant human PFKFB4 has a maximum velocity of $11.7 \pm 0.82 \mathrm{pmoles} /$ minute.}

In order to examine the bisphosphatase activity of PFKFB4, purified recombinant PFKFB4 protein was assayed in a bisphosphatase assay. This assay measures the bisphosphatase activity by incubating purified PFKFB4 enzyme with increasing concentrations of F-2,6-BP and other reactants and then removing aliquots from the reaction at various time points. These aliquots were then assayed for F-2,6-BP in a subsequent enzyme-coupled reaction. Using the reported bisphosphatase activity reported in the literature of the rat form of PFKFB4, we optimized the conditions of the reaction. Ultimately, 5 concentrations of F-2,6-BP in the range of $5-160 \mu \mathrm{M}$ were used to calculate the bisphosphatase activity of PFKFB4 (Figure 10).

From the data extrapolated from the bisphosphatase assay, the maximum velocity and Michaelis-Menten constant were determined. The maximum velocity $(11.7 \pm 0.82$ pmoles/minute $)$ and Michaelis-Menten constant $(18.2 \pm 4.7 \mu \mathrm{M})$ at a $95 \%$ confidence level. Therefore, the $\mathrm{K}_{\mathrm{cat}}$ for the bisphosphatase domain of PFKFB4 is calculated as $1.17 \pm 0.1 \mathrm{sec}^{-1}$. 
Figure 10. The bisphosphatase domain of PFKFB4 has a maximum velocity of $11.7 \pm 0.82 \mathrm{pmoles} / \mathrm{minute}$. Purified human recombinant PFKFB4 was assayed for bisphosphatase activity. For each reaction 10 $\mu \mathrm{g}$ of PFKFB4 was assayed with a range of substrate concentrations (5, $1040,80$, and $160 \mu \mathrm{M})$.

\section{Figure 10}

$M$ ic $h$ a e lis $-M$ e $n$ te $n$ :

P F K F B 4 P h o s $p h$ a ta s e A c tiv ity

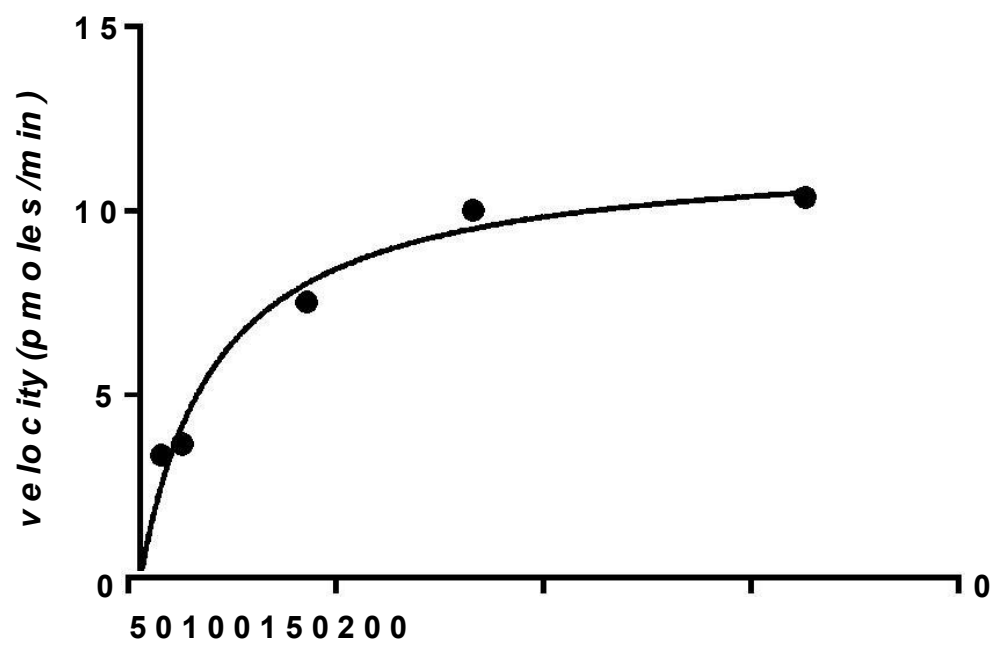

[Fructose-2,6-bisphosphate] $\mu \mathrm{M}$ 
The bisphosphatase domain of recombinant human PFKFB3 has a maximum velocity of $11.7 \pm 0.82 \mathrm{pmoles} /$ minute.

We next similarly assayed the bisphosphatase activity of PFKFB3 using purified recombinant PFKFB3 protein. From the data obtained from the bisphosphatase assay, the data points were fit to a Michaelis-Menten curve (figure 11) and the maximum velocity and Michaelis-Menten constant were determined. The maximum velocity was calculated to be $4.34 \pm 0.59$ pmoles/minute and Michaelis-Menten constant found to be $69.1 \pm 21.5 \mu \mathrm{M}$ (95\% confidence interval). The $\mathrm{K}_{\mathrm{cat}}$ for the bisphosphatase domain of PFKFB3 was calculated as $0.434 \pm 0.059 \mathrm{sec}^{-1}$. 
Figure 11.The bisphosphatase domain of PFKFB3 has a maximum velocity of $4.34 \pm 0.059$ pmoles/minute. Purified human recombinant PFKFB4 was assayed for bisphosphatase activity. For each reaction, 10 $\mu \mathrm{g}$ of PFKFB3 was assayed with increasing substrate concentrations (5, $1040,80$, and $160 \mu \mathrm{M})$.

Figure 11
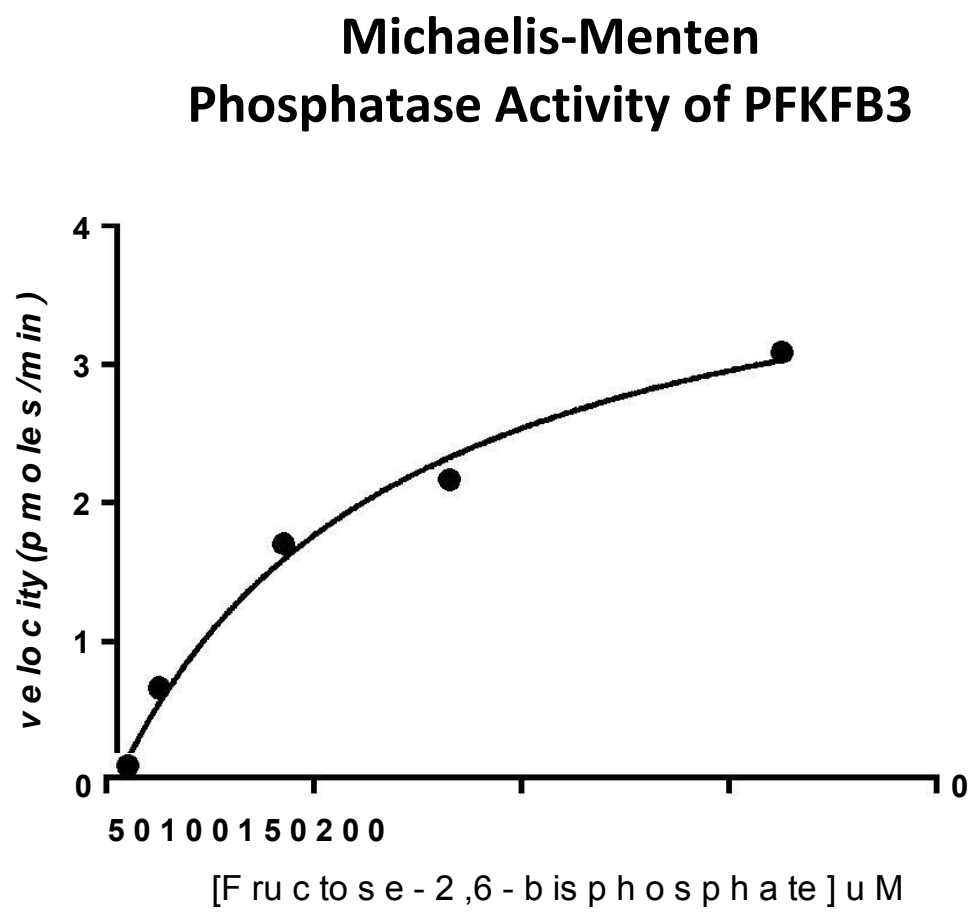


\section{DISCUSSION}

Members of the PFKFB family have two enzymatic activities. This dual function dictates the concentration of an important allosteric activator of glycolysis by interconverting F-6-P and F-2,6-BP. Previously, PFKFB3 has been thought to be the most important family member because of a sequence variation that diminishes its bisphosphatase activity allowing the enzyme to function almost exclusively as a kinase [9]. A variety of human cancer cell types show an over-expression of the PFKFB3 isoform, and, as a result, an increase in glycolytic flux to support their growth and energy requirements [40]. Recent data, however, indicate the PFKFB4 isoform may perform an equally critical function in cancer cells [20].

The members of the PFKFB family of enzymes have been demonstrated to have varied kinase:bisphosphatase ratios [21]. A higher kinase activity suggests that the enzyme's primary function is in the synthesis of F-2,6-BP, a major allosteric activator of PFK-1 and inhibitor of fructose-1,6-BP [21] and implies that it may perform an important function in cancer by increasing glycolysis to fulfill the energy requirements for the

growing tumor. While this principle has been exploited in drug development through targeting PFKFB3, the enzymatic activities and thus the relevance of PFKFB4 have yet to be fully explored.

To determine whether PFKFB4 functions predominantly as a kinase, we first sought to analyze its kinase activity. Using the published known rat PFKFB4 Michaelis-Menten constant of $85 \mu \mathrm{M}$ [23] as a starting 
point, we calculated kinase reaction rates using several substrate concentrations. Using this method, we calculated PFKFB4's maximum velocity (25.2 \pm 0.93 pmoles/minute) and Michaelis-Menten constant (356 $\pm 44.7 \mu \mathrm{M})$ at a $95 \%$ confidence level.

We similarly examined the kinase activity of the PFKFB3 isoform. The published Michaelis-Menten constant for PFKFB3 is $32 \mu \mathrm{M}$ [13] and we therefore varied the F6P concentrations from 1 to $80 \mu \mathrm{M}$ in order to calculate the maximum velocity (23.2 \pm 0.82 pmoles/minute $)$ and Michaelis-Menten constant (10.2 $\pm 1.5 \mu \mathrm{M})$.

Through our experiments, we determined the Kcat for the kinase domain of PFKFB4 to be $5.03 \mathrm{sec}^{-1}$ and to be $1.17 \mathrm{sec}^{-1}$ for the bisphosphatase domain. Therefore the kinase activity of human PFKFB4 is 4.3 times that of the bisphosphatase activity (see table below). This ratio is very similar to the reported ratio for rat PFKFB4 that was reported as 4.1:1 in the literature [23]. We also calculated the Kcat of the kinase and bisphosphatase domains of PFKFB3 which were determined to be 46.5 $\mathrm{sec}^{-1}$ and $.434 \mathrm{sec}^{-1}$ respectively, giving human recombinant PFKFB3 a kinase:bisphosphatase ratio of 107:1 (see table below).

\begin{tabular}{|l|c|c|}
\hline Isoform & PFKFB4 & PFKFB3 \\
\hline Kinase Activity $\left(\mathrm{sec}^{-1}\right)$ & 5.03 & 46.5 \\
\hline Phosphatase Activity $\left(\mathrm{sec}^{-1}\right)$ & 1.17 & .434 \\
\hline Kinase:phosphatase ratio $\left(\mathrm{sec}^{-1}\right)$ & $4.3: 1$ & $107: 1$ \\
\hline
\end{tabular}


Our conclusion that PFKFB4 functions primarily as a kinase is further supported by the intracellular concentrations of F-6-P and F-2,6-BP. Since the concentration of F-6-P, the substrate for the kinase domain, is $>10,000$ fold the concentration of $\mathrm{F}-2,6-\mathrm{BP}$, the substrate for the phosphatase domain (e.g. MCF-7 cells: F6P, 50 $\pm 24 \mathrm{nmol} / \mathrm{mg}$ protein [48]; F2,6BP, $2.97 \pm .21 \mathrm{pmol} / \mathrm{mg}$ protein [49]), then the kinase activity should be dominant.

Since 2012, the data supporting the development of cancer therapeutics targeting PFKFB4 has been significantly expanded. PFKFB4 has been found to be critical for cancer cell survival in gliomas and prostate cancer and breast cancer, colon cancer, and lung cancer lines [18-20]. Furthermore, in 2014 Chesney, Clark et al. showed that PFKFB4 over-expression in 6 different cancer cell lines increased F-2,6-BP production, ATP production, and glycolysis [20]. Although these studies indicate that PFKFB4 functions predominantly as a kinase, a direct measurement of human PFKFB4 recombinant protein had not previously been conducted. Therefore, this present study includes a direct measurement of the kinase and bisphosphatase activities of PFKFB4. By determining biochemically that the kinase domain has a 4.3 times greater activity than the bisphosphatase domain, our data strongly support the development of small molecule inhibitors targeting the kinase domain of PFKFB4 to decrease the intracellular concentration of F-2,6-BP and reduce the glycolytic flux in cancer cells. 


\title{
CHAPTER VI
}

\author{
PFKFB4 IS INHIBITED BY THE SMALL MOLECULE INHIBITOR 5MPN \\ AND NOT INHIBITED BY COMPOUNDS TARGETED AGAINST PFKFB3
}

\section{INTRODUCTION}

A common characteristic of most human cancers is an increased glucose uptake and metabolism due to genetic alterations that increase the expression of many glycolytic enzymes [5]. The PFKFB family of enzymes (PFKFB1-4) synthesize F-2,6-BP which activates PFK-1, a key glycolytic enzyme that catalyzes the conversion of fructose-6-phosphate to fructose-1,6-bisphosphate.

Of the PFKFB family, the PFKFB3 family member has been extensively studied. Research has shown that PFKFB3 expression is increased by hypoxia and a direct transcriptional target of HIF1 $\alpha$ [38]. Not only is the expression level of PFKFB3 increased in most tumor types [39], but the heterozygous deletion of the Pfkfb3 gene has been shown to decrease levels of F-2,6-BP, glucose uptake, and glycolytic flux to lactate [40]. These data along with other studies demonstrated that PFKFB3 is a rational target for the development of anticancer therapies and a small 
molecule inhibitor of PFKFB3 (3-(3-pyridinyl)-1-(4-pyridinyl)-2-propen-1one, termed 3PO) was developed in 2008 [41]. Subsequently, derivatives of $3 \mathrm{PO}$ were screened from which one compound was used for further testing, 1-(4-pyridinyl)-3-(2-quinolinyl)-2-propen-1-one (PFK15).

PFK15 was tested in vivo and in vitro and was shown to cause apoptosis in a leukemia cell line, have superior pharmacokinetic properties to $3 \mathrm{PO}$, decrease growth in a Lewis Lung Carcinoma xenograft model and suppress ${ }^{18}$ F-FDG uptake by LLC xenografts [41]. Following the investigation of PFK15, another derivative of 3PO has now been developed, termed PFK158. This derivative is more potent than 3PO, has improved pharmacokinetic properties, and causes an approximately $80 \%$ inhibition of tumor growth in several xenograft tumor models [42]. PFK158 is currently being tested in a phase 1 clinical trial in advanced cancer patients (clinicaltrials.gov \#NCT02044861).

Expression of the PFKFB4 family member has also been shown to be increased in multiple tumors, induced significantly by hypoxia [20] and critical for cancer cell survival. Genomic and siRNA-mediated deletion of PKFB4 has been found to cause a decrease in F2,6 BP, proliferation and lead to increased apoptosis. These data provide rationale for the development of inhibitors targeted against PFKFB4 and recently, a novel small molecule inhibitor of the PFKFB4 enzyme has been identified through in silico screening (termed 5MPN). 5MPN decreases F-2,6-BP production, glucose uptake, and cell proliferation in vitro and daily 5MPN 
administration in a mouse xenograft model has been found to decrease tumor growth in vivo.

The co-expression of the PFKFB3 and PFKFB4 isoforms in multiple tumors makes it likely that targeting both the PFKFB3 and PFKFB4 isoforms may be required in order to adequately suppress the glycolytic flux that is characteristic of tumor cell metabolism. Although it is clear that PFK15 and PFK158 target PFKFB3 and result in decreased tumor growth in vivo through a decrease in glucose uptake, it remains uncertain as to how specific these inhibitors are for PFKFB3 over the other PFKFB family members. Therefore, our objective is to assess the selectivity of the known inhibitors of PFKFB3, PFK15 and PFK158, as well as investigate inhibition of PFKFB4 and PFKFB3 by a novel PFKFB4 small molecule inhibitor, 5MPN.

We sought to examine the effect of 5MPN on PFKFB4 activity in a kinase assay and additionally, examine the effect of the PFKFB3 inhibitors, PFK15 and PFK158, on both the PFKFB4 and PFKFB3 enzymes in order to determine their specificity for their targets and their effectiveness at blocking F-2,6-BP production. In addition to an examination of the effects of these inhibitors on the production of F-2,6$\mathrm{BP}$, we chose to also examine the effect of these selective kinase inhibitors for PFKFB3 and PFKFB4 on the activity of the bisphosphatase in order to allow a more accurate measurement of the bisphosphatase activity and thus of the kinase to bisphosphatase ratio. 


\section{RESULTS}

\section{PFK15 and PFK158 competitively inhibit PFKFB3 better than PFKFB4}

Several published inhibitors have been developed to target PFKFB3. We chose to examine PFK15 and PFK158 and assessed their effect on the activity of PFKFB3 and PFKFB4 with the aim of determining whether these inhibitors are more selective for PFKFB3 than PFKFB4. To analyze the data, Sigmaplot ranks the possible models of inhibition, competitive inhibition, non-competitive inhibition, and mixed inhibition, according to their coefficient of determination $\left(\mathrm{R}^{2}\right)$ and Akaiki Information Criterion (AIC) values in order to select the model that best fit the data [43]. AIC is a technique that measures the goodness of fit of a statistical model and selects a model from a set of candidate models. The chosen model is the one that is expected to minimize the difference between the model and the truth. Given a data set, several possible models may be ranked according to their $\mathrm{AIC}$ value, and the model giving the lowest $\mathrm{AIC}$ value is selected as having the best fit for the data set [43]. Using the best model, we then compared the inhibition constants $\left(\mathrm{K}_{\mathrm{i}}\right)$ to determine whether the drug better inhibits PFKFB3 or PFKFB4.

Since 3PO, the parent compound to PFK15 and PFK158, is reported to show a mixed inhibition model, we hypothesized that PFK15 and PFK158 would inhibit through the same mechanism. We examined increasing concentrations of PFK15 $(100 \mathrm{nM}, 1 \mu \mathrm{M}$, and $10 \mu \mathrm{M})$ in the kinase reaction with several substrate concentrations $(5,10,40,80$, and 
$320 \mu \mathrm{M})$ and $500 \mathrm{ng}$ of PFKFB3 and selected the best inhibition model. First, the models were ranked according to their $R^{2}$ values. The competitive and mixed inhibition models shared the same $R^{2}$ value of 0.93 which was higher than the $R^{2}$ value for noncompetitive inhibition model. Next, the AIC values were compared to select whether competitive or mixed inhibition better fit the data set. Given that the preferred model is the one with the lower AIC value, and that the competitive model had a value of 2.54 and the mixed model had a value of 3.42 , then it is suggested that PFK15 inhibits PFKFB3 through a competitive inhibition model (Figures 12-13). Therefore, using the competitive inhibition model the $\mathrm{K}_{\mathrm{i}}$ of PFK15 for PFKFB3 is $2.14 \pm 0.59 \mu \mathrm{M}$. 
Figure 12. PFK15 competitively inhibits PFKFB3 and the $K I$ is $2.1 \pm$

0.59. After comparing possible models of the interaction between PFK15 and PFKB3, competitive inhibition was concluded as the model of inhibition because it has the highest $R^{2}$ value (0.93) and lowest AIC value (2.54). Using this model, the $K_{i}$ value of PFK15 for PFKFB3 is $2.14 \pm 0.59$.

Figure 12

Michaelis-Menten

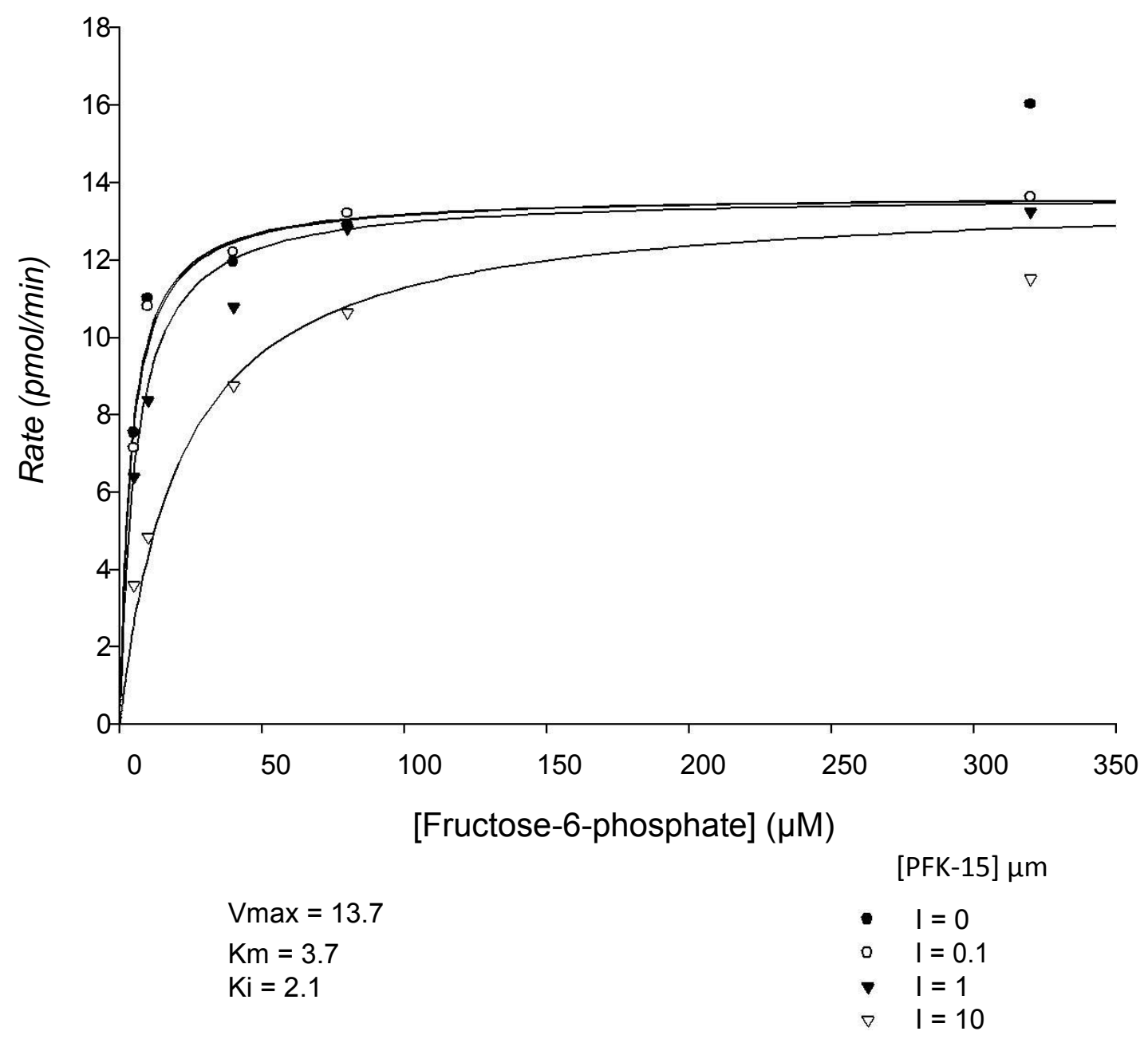


Figure 13. The Lineweaver-Burk plot also suggests that PFK15 competitively inhibits PFKFB3. The data points were also fit to a Lineweaver-Burk plot. This plot also suggests competitive inhibition as the model of inhibition of PFK15 for PFKFB3.

Figure 13

Lineweaver-Burk

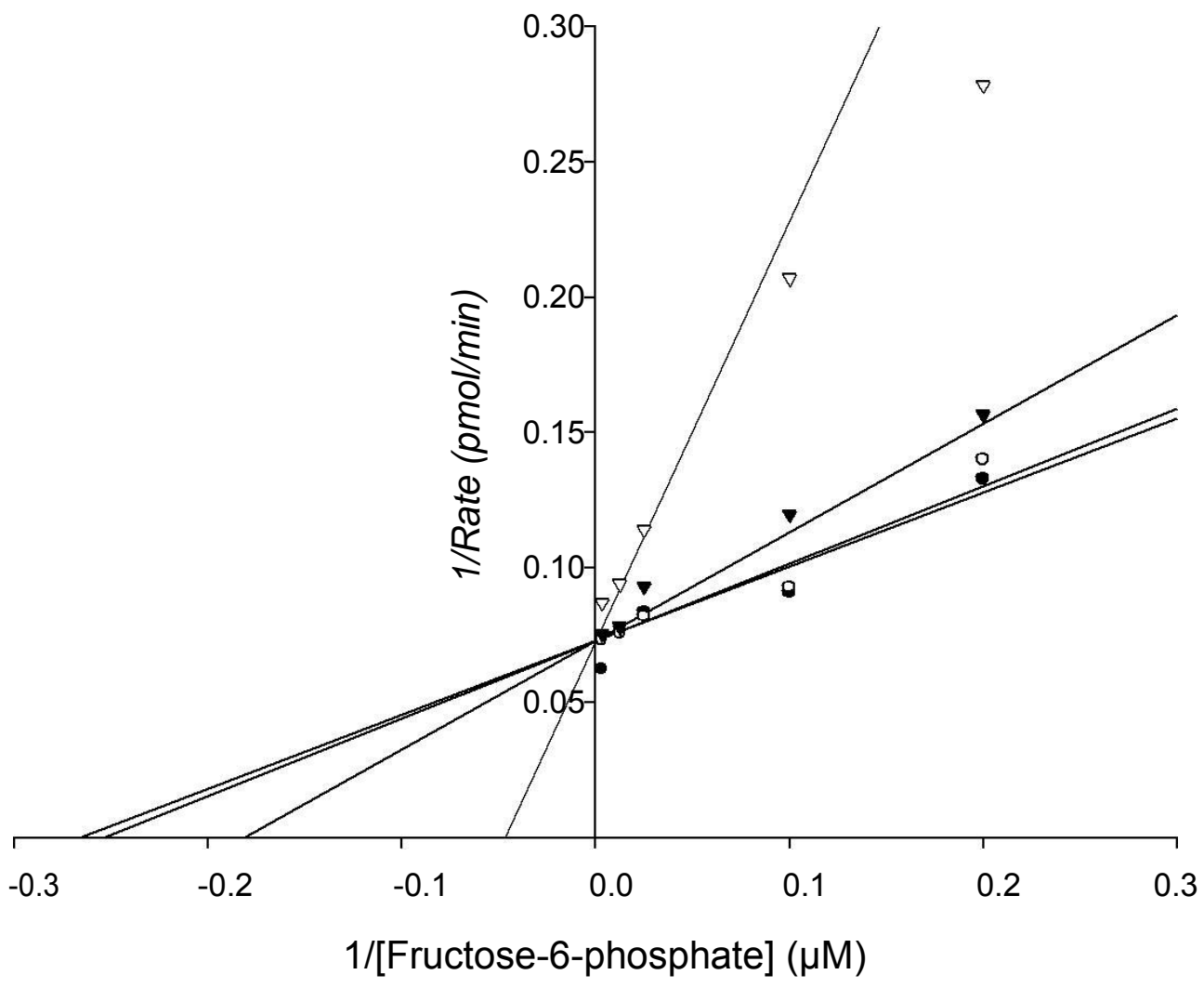

$V \max =13.7$

$\mathrm{Km}=3.7$

$\mathrm{Ki}=2.1$

[PFK-15] $\mu \mathrm{m}$

- $\mathrm{I}=0$

$\circ \quad I=0.1$

$\checkmark \quad \mathrm{I}=1$

$\nabla \quad \mathrm{I}=10$ 
Similarly, we used the same concentrations of PFK15 to examine its affinity for PFKFB4 in the same kinase reactions we used to assay its kinase activity. Also to the kinase reaction, several concentrations of substrate $(42.5 \mu \mathrm{M}, 85 \mu \mathrm{M}, 170 \mu \mathrm{M}, 340 \mu \mathrm{M}$, and $680 \mu \mathrm{M})$ and $5 \mu \mathrm{g}$ of PFKFB4 were added. Again, we ranked the different models to select the one that best fits the data set. The noncompetitive inhibition model had the lowest $R^{2}$ value $(0.89)$ and competitive and mixed inhibition shared the same, higher $\mathrm{R}^{2}$ value (0.91). The AIC values were used to further rank the competitive and mixed inhibition models, which were 57.4 and 61.0 respectively. Like PFKFB3, the AIC value for competitive inhibition is lower than that of the mixed inhibition model and is therefore the model that best fits the data set (Figures 14-15). According to the competitive model of inhibition, the $K_{i}$ value for PFK15 for PFKFB4 is $10.8 \pm 5.1$. 
Figure 14. PFK15 competitively inhibits PFKFB4. After comparing possible models of the interaction between PFK15 and PFKB4, the competitive inhibition model had the highest $R^{2}$ value $(0.91)$ and lowest AIC value (57.4). Therefore, competitive inhibition was concluded as the best model of the interaction between PFKFB4 and PFK-15. Using this model, the $\mathrm{K}_{\mathrm{i}}$ value of PFK15 for PFKFB5 is $10.8 \pm 5.1$.

\section{Figure 14}

\section{Michaelis-Menten}

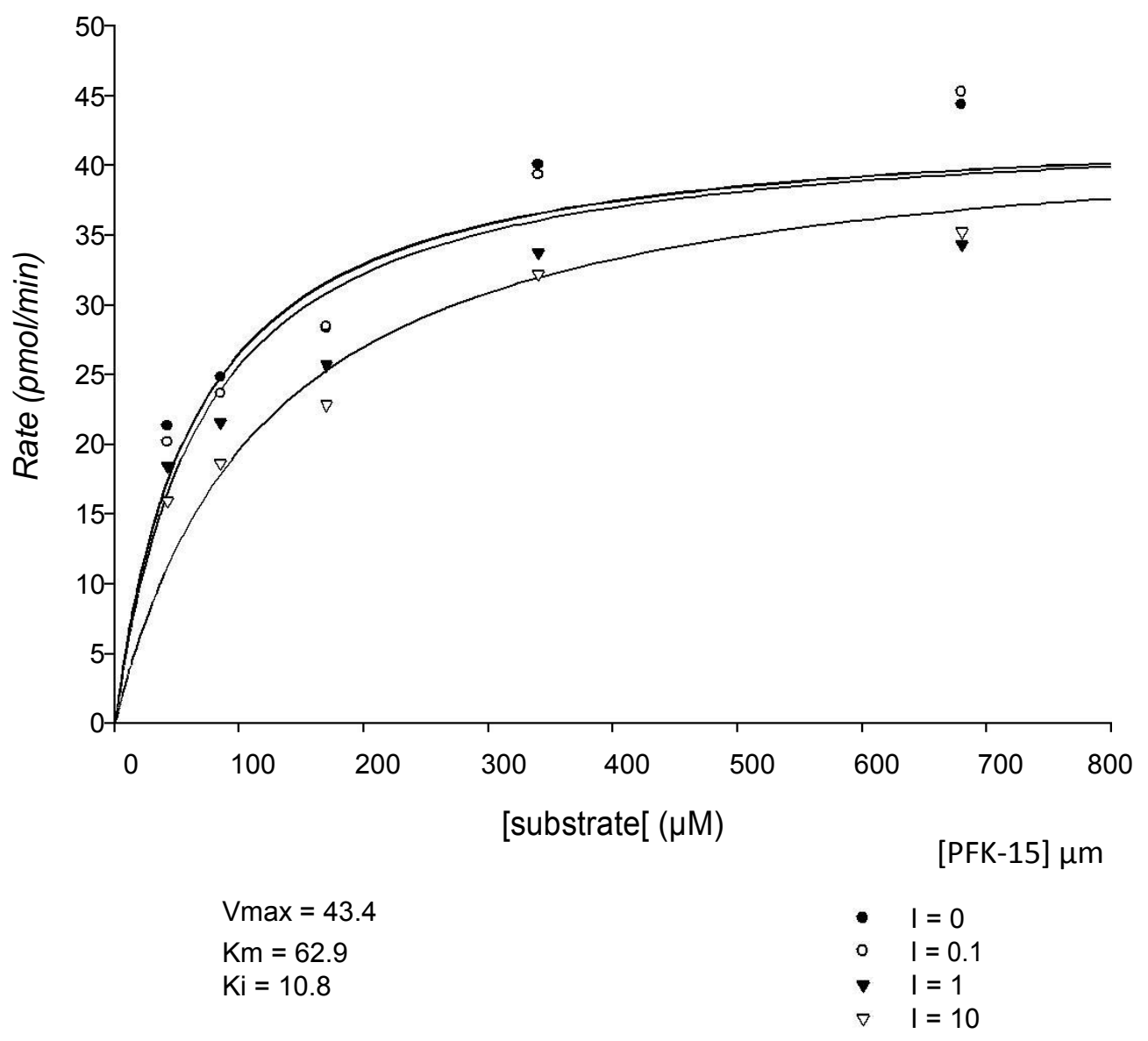


Figure 15. The Lineweaver-Burk plot also suggests that PFK15 competitively inhibits PFKFB4. The data points from the kinetic assay data used for the Michaelis-Menten curve were also fit to a LineweaverBurk plot. This plot also suggests competitive inhibition as the model of inhibition of PFK15 for PFKFB4.

\section{Figure 15}

\section{Lineweaver-Burk}

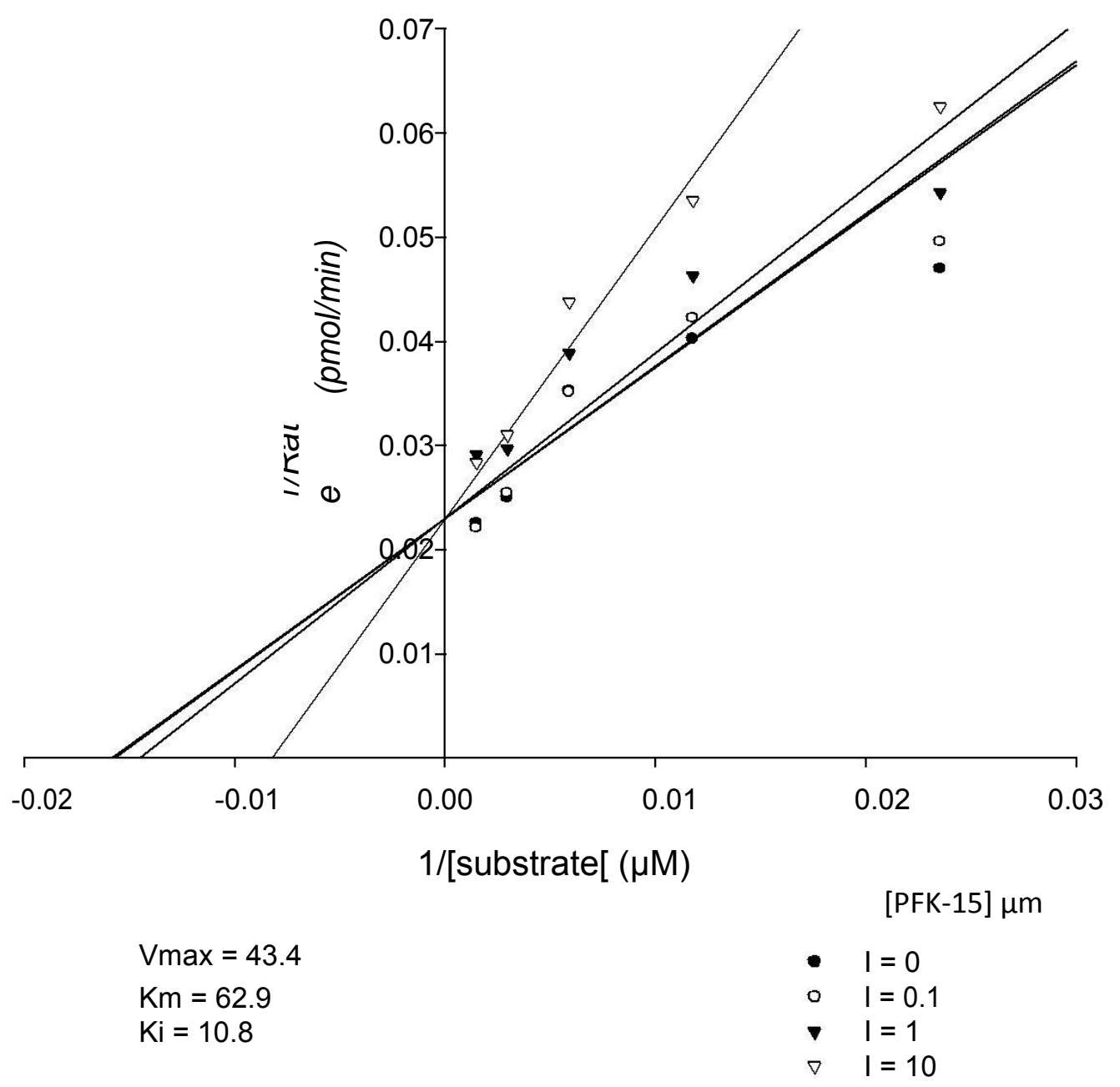


Since PFK158 is the PFKFB3 inhibitor that is currently being using in clinical trials, we chose to also examine the effect of PFK158 on PFKFB3 and PFKFB4. We used the exact methods used when analyzing the inhibition of PFK15 for PFKFB3 and PFKFB4. Mixed and competitive inhibition had and equal $R^{2}$ value $(0.87)$ that was higher than noncompetitive inhibition, but the AIC value for the competitive inhibition model was lower than the value for mixed inhibition; 38.0 and 41.6 respectively. The data suggested that the competitive inhibition model best fit the data (Figures 16-17) and therefore, the KI value for PFK158 for PFKFB3 is $10.1 \pm 6.0$. For PFKFB4, the competitive, noncompetitive, and mixed models all shared the same $\mathrm{R}^{2}$ value, 0.99 . However, the competitive inhibition model had the lowest AIC value, 41.5 than the other noncompetitive and mixed inhibition which were 44.8 and 45.1 respectively. Thus, it is suggested that the competitive inhibition model best fit the PFKFB4 data (Figures 18-19) and the Ki value for PFKF158 inhibiting PFKFB4 is $34.2 \pm 14.6$. 
Figure 16. PFK158 competitively inhibits PFKFB3 and the $K_{I}$ is $10.1 \pm$

6.0. Three models of inhibition, competitive, non-competitive, and mixed inhibition, were compared for the interaction between PFK158 and PFKB3. Competitive inhibition was concluded as the model of inhibition because it has the highest $R^{2}$ value (0.87) and lowest AIC value (38). Using this model, the Ki value of PFK158 for PFKFB3 is $10.1 \pm 6.0$.

\section{Figure 16}

Michaelis-Menten

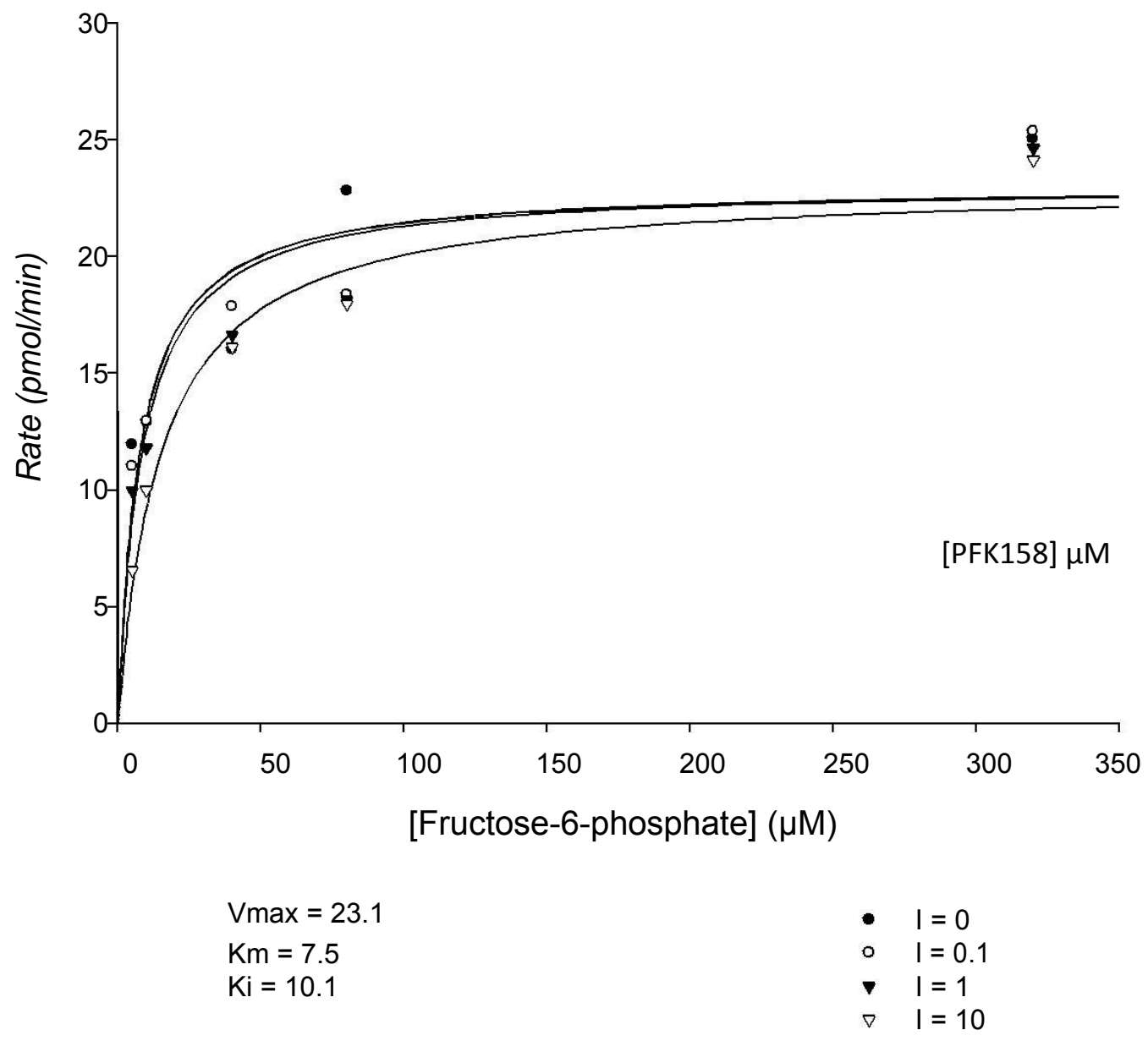


Figure 17. The Lineweaver-Burk plot also suggests that PFK158 competitively inhibits PFKFB3. The data points from the kinetic assay of PFKFB3 with PFK158 were also fit to a Lineweaver-Burk plot.

\section{Figure 17}

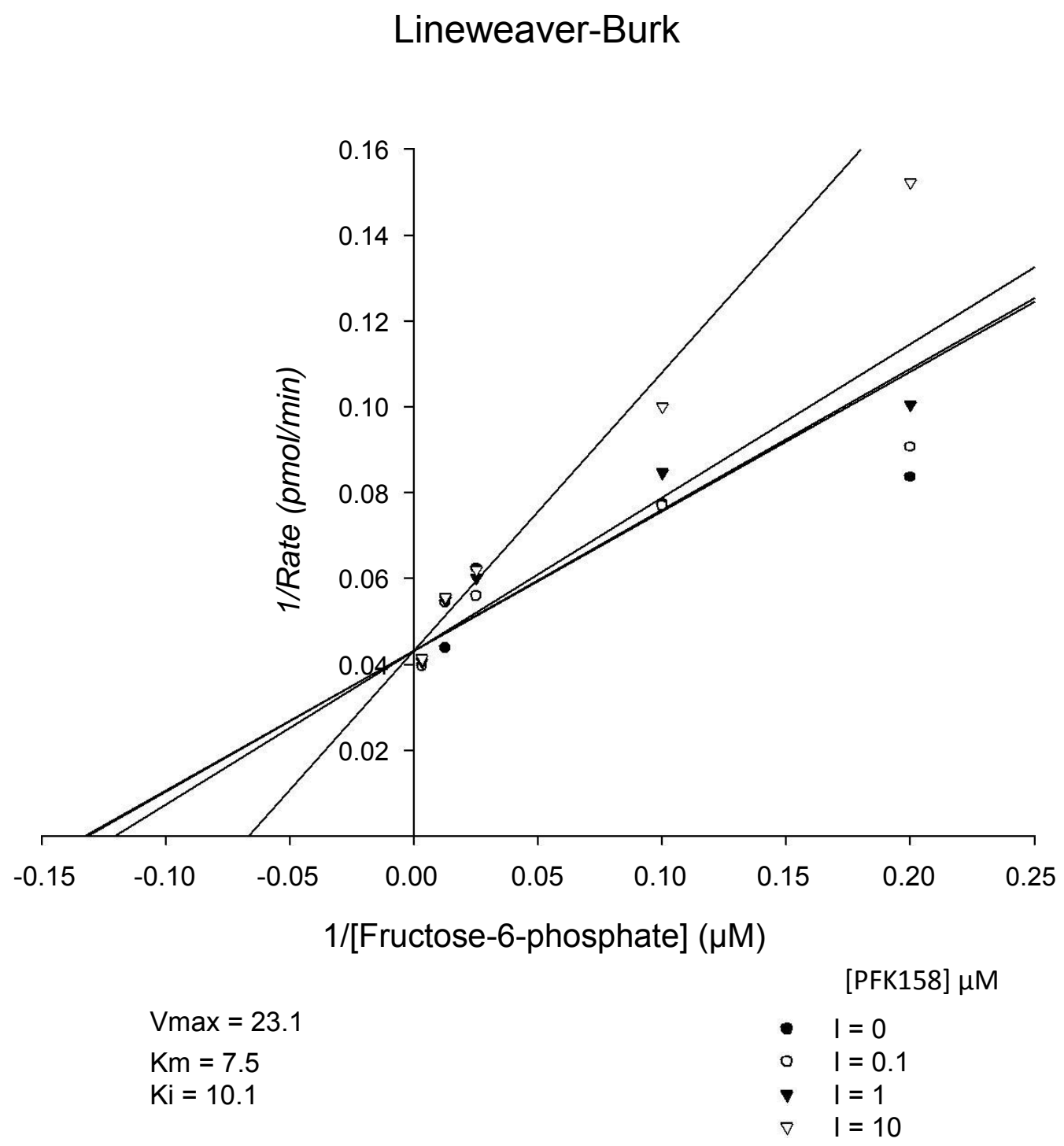


Figure 18. PFK158 competitively inhibits PFKFB4, however, with a KI value of $34.2 \pm 14.6$. Of the models of inhibition used to analyze the kinetic data generated from the interaction between PFK158 and PFKFB4, competitive inhibition best models the relationship. Competitive inhibition has both the highest $R^{2}$ value (0.99) and lowest AIC value (38). Using this model, the $\mathrm{K}_{i}$ value of PFK158 for PFKFB4 is $34.2 \pm 14.6$.

\section{Figure 18}

Michaelis-Menten

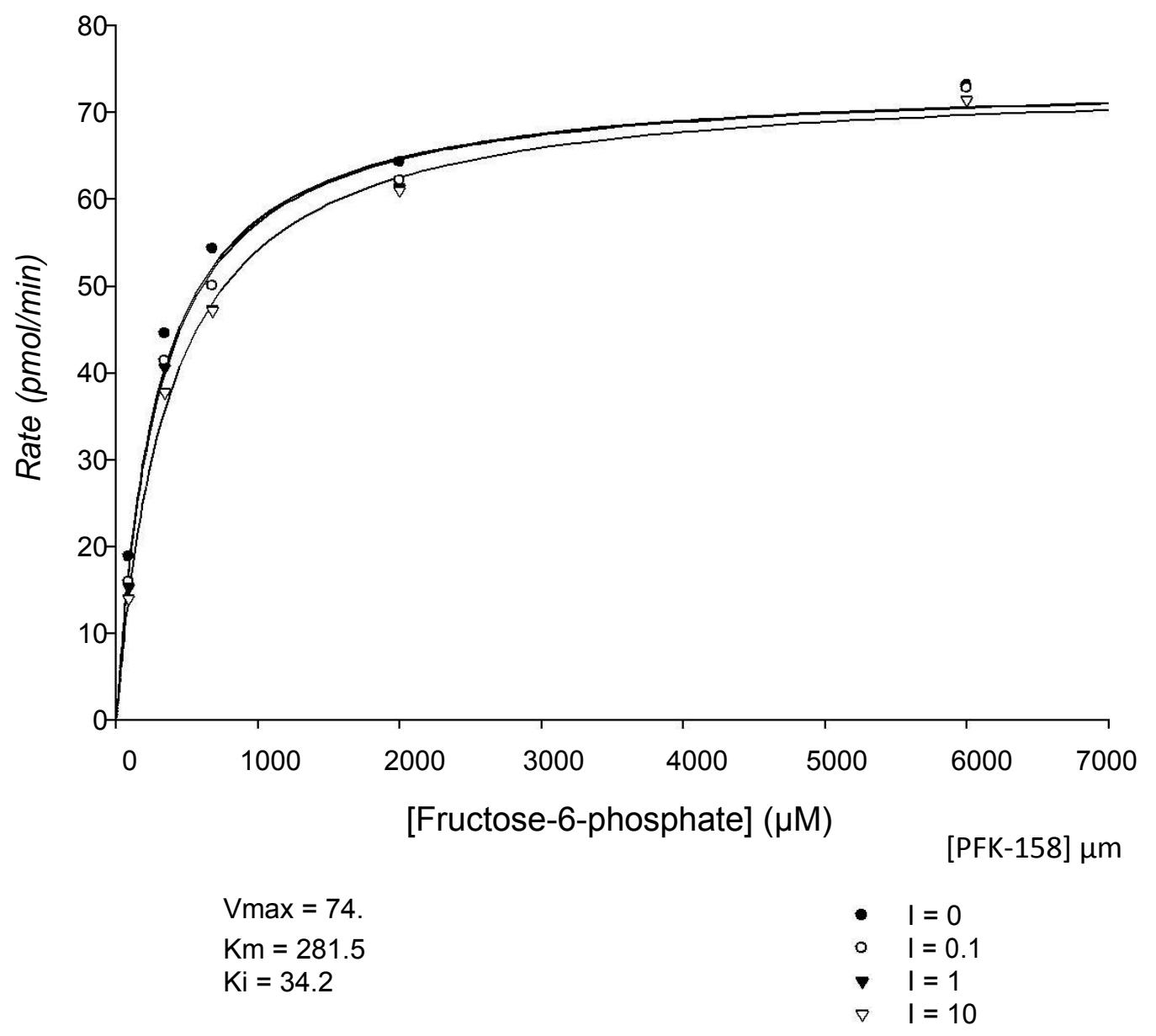


Figure 19: PFK158 blocks PFKFB4 through competitive inhibition. A Lineweaver-Burk plot was also used to model the kinetic data from the kinetic assay of PFK158 and PFKFB4.

Figure 19

\section{Lineweaver-Burk}

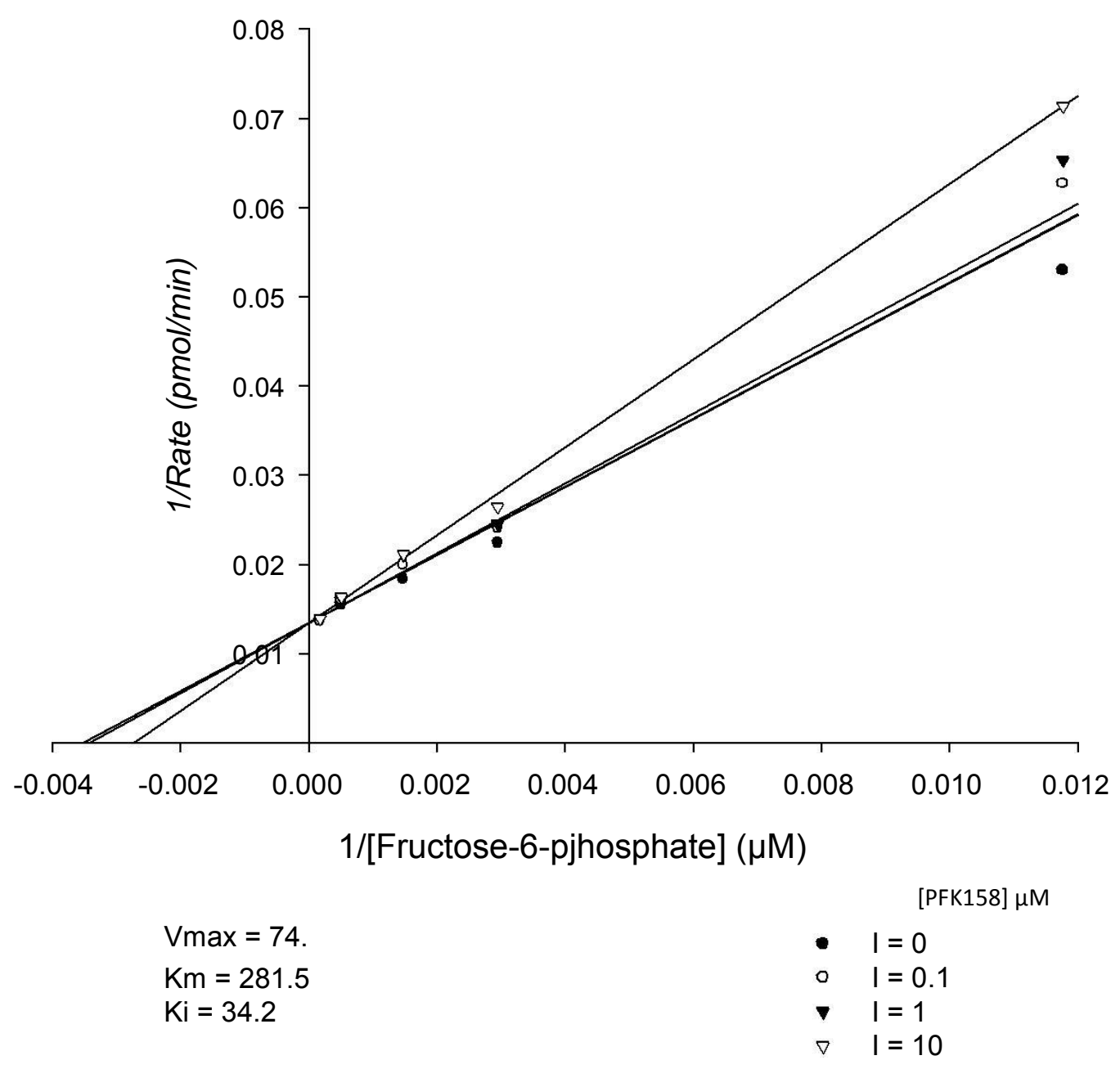


Although the PFKFB3 inhibitors, PFK15 and PFK158, also target PFKFB4, they do so with a much lower affinity. Therefore, we next elected to look at a novel PFKFB4 inhibitor, 5MPN to examine its effect on PFKFB4 and determine if it also inhibits PFKFB3. Different concentrations of $5 \mathrm{MPN}(0 \mu \mathrm{M}, 100 \mathrm{nM}, 1 \mu \mathrm{M}$, and $10 \mu \mathrm{M})$ were added to the kinase reaction with PFKFB4 in which we used $5 \mu \mathrm{g}$ of PFKFB4 and 5 concentrations of fructose-6-phosphate $(10 \mu \mathrm{M}, 85 \mu \mathrm{M}, 340 \mu \mathrm{M}, 680 \mu \mathrm{M}$, and $2 \mathrm{mM}$ ). Three models of inhibition were then ranked according to their $R^{2}$ values. Competitive and mixed inhibition both had an $R^{2}$ value of 0.98 , while noncompetitive had a lower value of 0.97 . The competitive model had the lower AIC value (-12.4) than the mixed model (-8.8). Accordingly, the data suggests that the competitive inhibition is the best model (figures 20-21) and the $K_{i}$ value for $5-M P N$ for PFKFB4 is $8.6 \pm 1.9$. However, when we added the same concentrations of $5 \mathrm{MPN}$ to the kinase reaction with PFKFB3, the error was so high with each of the three models that the data was unable to be interpreted suggesting that 5MPN does not inhibit PFKFB3 (Figure 22). 
Figure 20. 5MPN competitively inhibits PFKFB4, and with a $K_{I}$ value of $8.6 \pm 1.9$, best inhibits PFKFB4. An inhibitor designed to selectively inhibit the substrate binding site of PFKFB4 was analyzed to determine the mode of inhibition. Of the models of inhibition, competitive inhibition best models the relationship between 5-MPN and PFKFB4. Competitive inhibition has both the highest $R^{2}$ value (0.98) and lowest AIC value (12.4). Using this model, the $K_{i}$ value of $5 M P N$ for PFKFB4 is $8.6 \pm 1.9$.

Figure 20

\section{Michaelis-Menten}

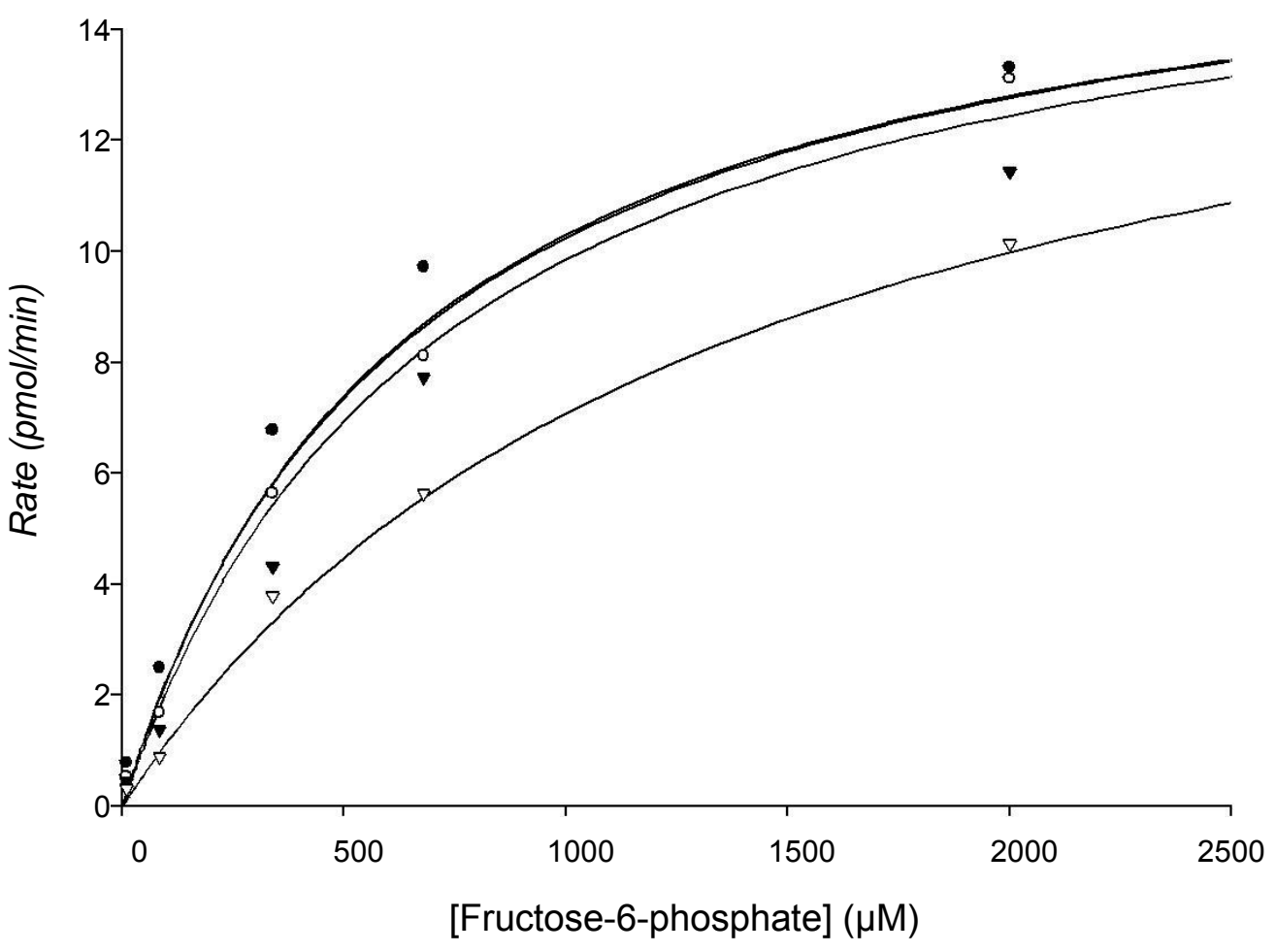

$V \max =16.9$

$\mathrm{Km}=642$.

$\mathrm{Ki}=8.6$

$\begin{array}{ll}- & I=0 \\ \circ \quad & I=[5 M P N] \mu m \\ \nabla \quad I & = \\ \nabla \quad & I=10\end{array}$ 
Figure 21. The Lineweaver-Burk plot also suggests competitive inhibition for the interaction between PFKFB4 and 5-MPN. A Lineweaver-Burke plot was also used to model the kinetic data from the kinetic assay of 5MPN and PFKFB4.

Figure 21

\section{Lineweaver-Burk}

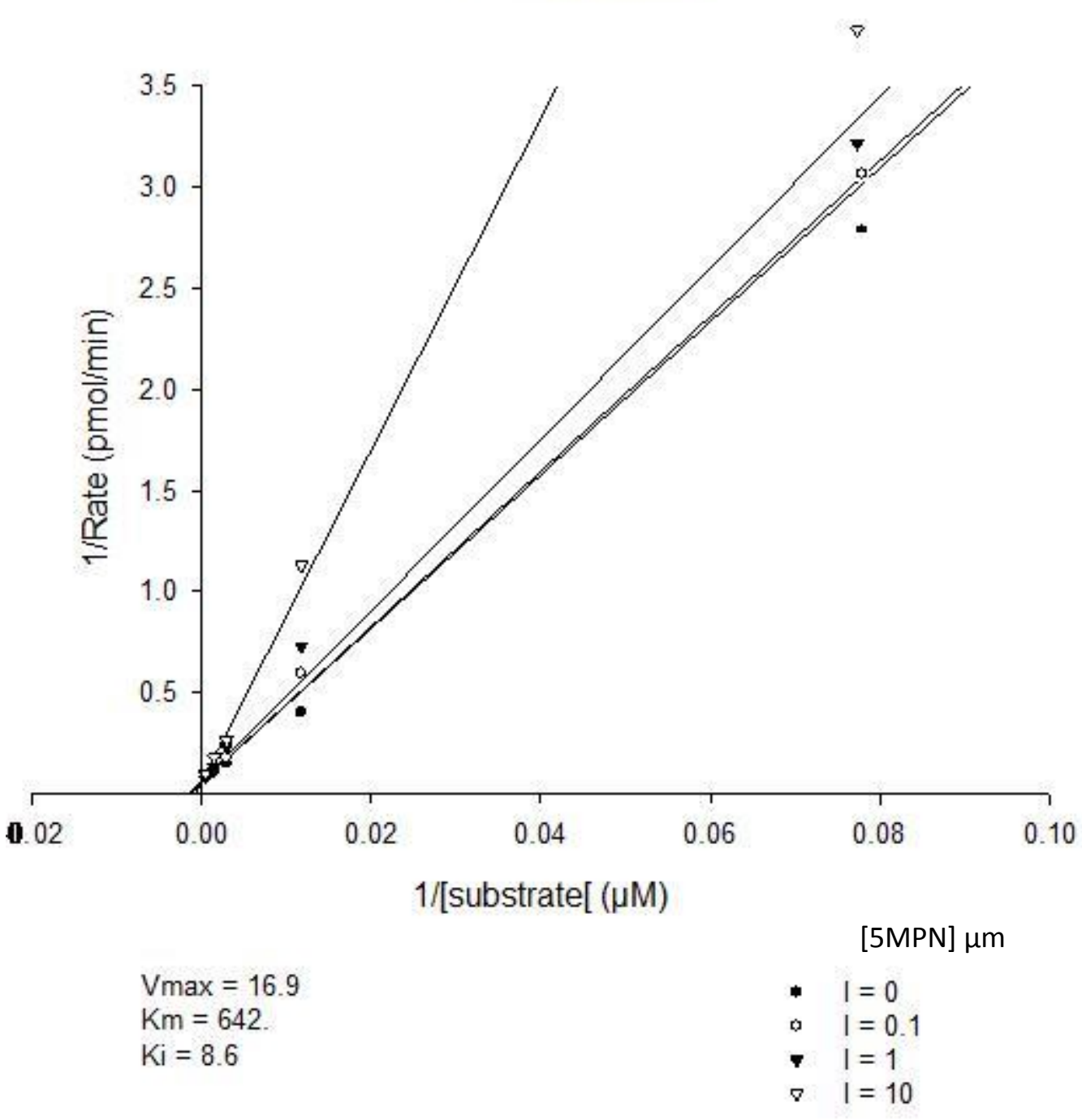


Figure 22. 5MPN does not inhibit PFKFB3. An inhibitor designed to selectively inhibit the substrate binding site of PFKFB4 was also analyzed to determine its specificity for the PFKFB3 isoform. The Michaelis-Menten plot indicates that 5MPN does not target the PFKFB3 isoform.

Figure 22

\section{Michaelis-Menten}

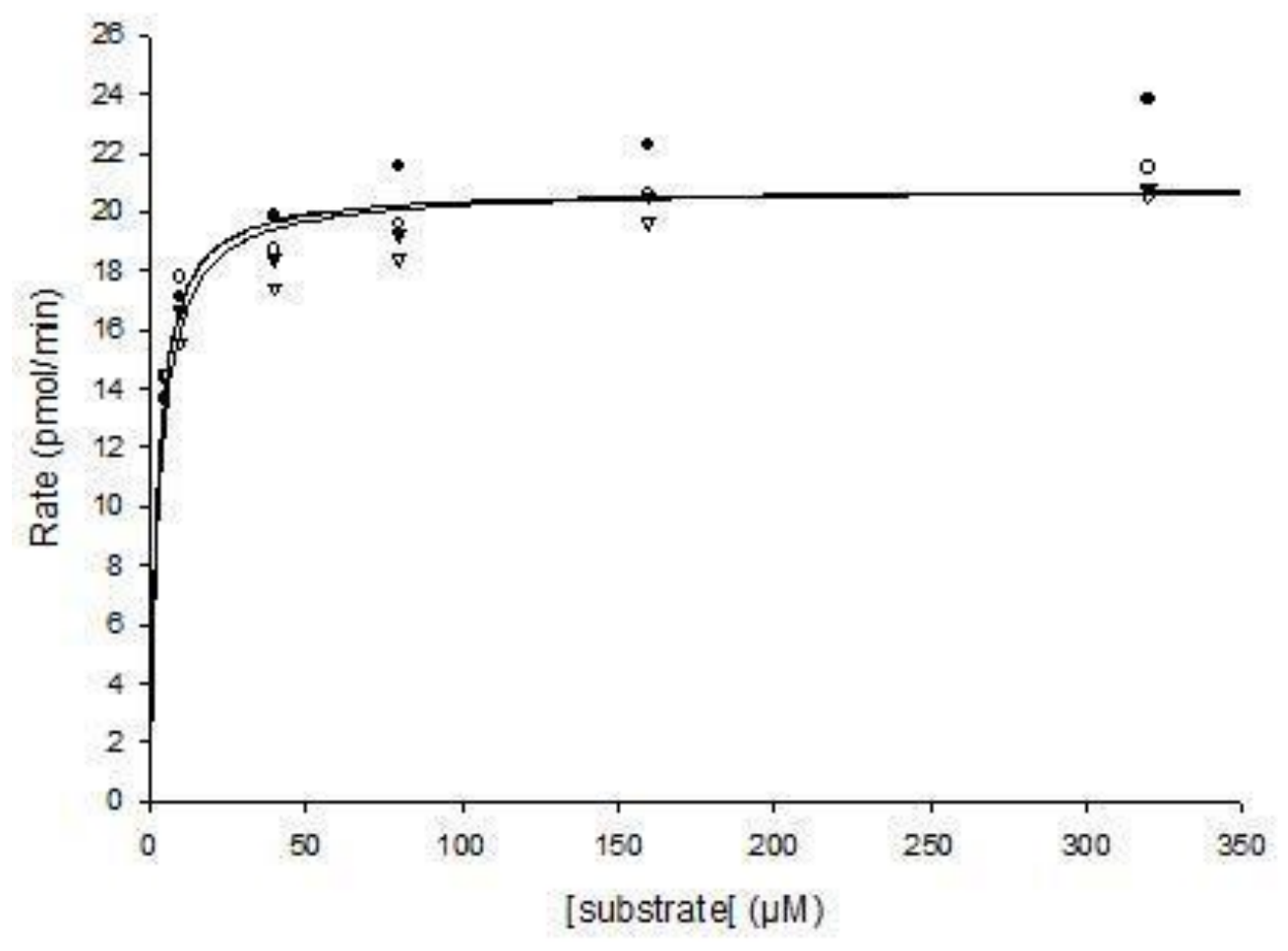

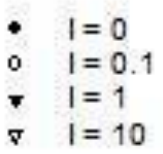


Adding kinase inhibitors to the bisphosphatase reactions increased the $\mathrm{V}_{\max }$ and $\mathrm{K}_{\text {cat }}$ for the bisphosphatase activities of both PFKFB3 and PFKFB4.

By previously testing the specificity of the PFKFB3 and PFKFB4 inhibitors, we were able to identify that PFK-15 best inhibits PFKFB3 and 5MPN best inhibits PFKFB4. Therefore, we added the highest concentrations $(10 \mu \mathrm{M})$ of drug to the bisphosphatase reactions in order to more accurately calculate the bisphosphatase reactions and therefore the kinase to bisphosphatase ratios for both PFKFB4 and PFKFB3. For PFKFB4 we followed the some procedures previously outlined, except that we added 5MPN to the bisphosphatase reaction (Figure 23). The reaction rates for each substrate concentration curve fits the Michaelis-Menten equation at an $R^{2}$ value of 0.99 As a result, the Vmax changed to $19.5 \pm$ 1.0 pmoles/second from $11.7 \pm 0.8$ pmoles/second and the $K_{c a t}$ changed to $1.9 \pm 0.1 \mathrm{sec}^{-1}$ from $1.17 \pm 0.1 \mathrm{sec}^{-1}$. 
Figure 23. The addition of 5-MPN to the bisphosphatase assay for PFKFB4 increased the $V_{\max }$ and $K_{\text {cat }}$. Inhibiting the kinase domain of PFKFB4 through the addition of $10 \mu \mathrm{M}$ of 5-MPN to the bisphosphatase reaction increased the $V_{\max }$ calculation to $19.5 \pm 1.0$ from $11.7 \pm 0.8$ pmoles/sec and changed the $K_{c}$ cat calculation from $1.17 \pm 0.1 \mathrm{sec}^{-1}$ to 1.9 $\pm 0.1 \mathrm{sec}^{-1}$.

\section{Figure 23}

$M$ ic $\mathbf{h}$ a e lis $-M$ e $n$ te $n$ :

P h os ph a ta s e A c tiv ity of PFK F B $4+5-M$ P N

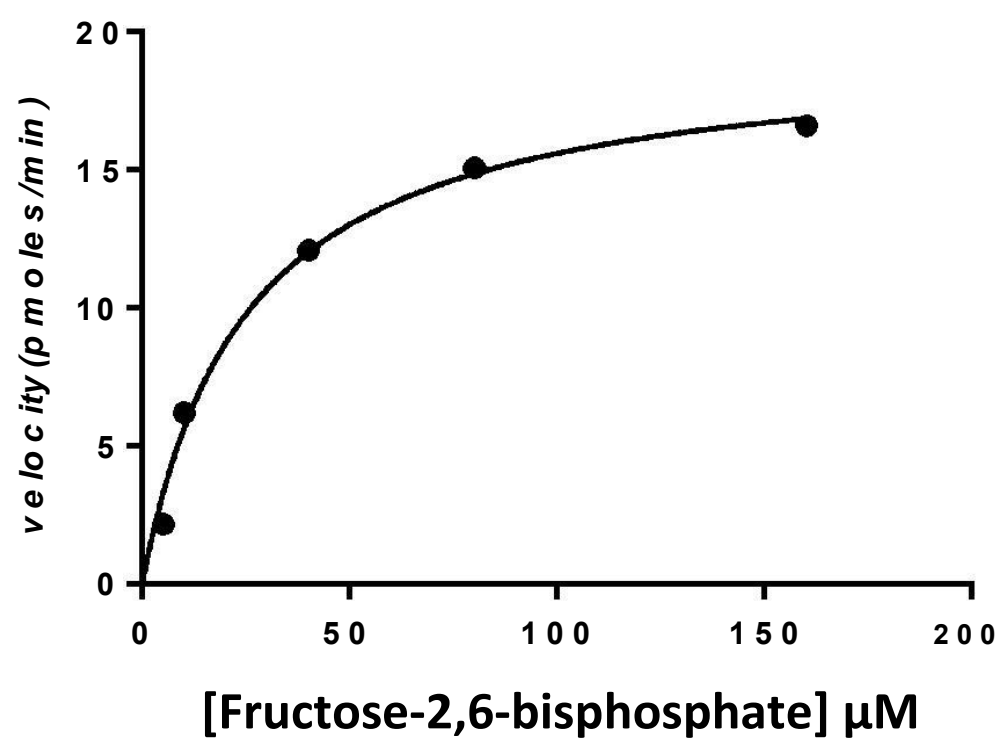


Subsequently, we repeated the bisphosphatase reaction for PFKFB3 in the same manner as previously described, however, we added PFK15 to the reaction. We chose PFK15 instead PFK158 because it has a higher $\mathrm{K}_{\mathrm{i}}$ value and therefore inhibits the kinase activity of PFKFB3 more efficiently. The calculated velocities for each substrate concentration adequately fit the Michaelis-Menten curve $\left(R^{2}=0.98\right.$, Figure 24). The addition of PFK15 to the reaction increased the $V_{\max }$ calculation to $5.6 \pm 0.4$ from $4.3 \pm 0.1$ and changed the $\mathrm{K}_{\text {cat }}$ calculation from $0.43 \pm 0.1 \mathrm{sec}^{-1}$ to $.56 \pm 0.04 \mathrm{sec}^{-1}$ 
Figure 24. The addition of PFK15 to the bisphosphatase assay for PFKFB3 increased the $\mathbf{V}_{\max }$ and $\mathrm{K}_{\text {cat. }}$ Inhibiting the kinase domain of PFKFB4 thought the addition of $10 \mu \mathrm{M}$ of PFK15 to the reaction increased the $V_{\max }$ calculation to $5.6 \pm 0.4$ from $4.3 \pm 0.1$ and changed the $\mathrm{K}_{\text {cat }}$ calculation from $0.43 \pm 0.1 \mathrm{sec}^{-1}$ to $.56 \pm 0.04 \mathrm{sec}^{-1}$.

Figure 24

\section{Michaelis-Menten \\ Phosphatase Activity of PFKFB3 +PFK15}

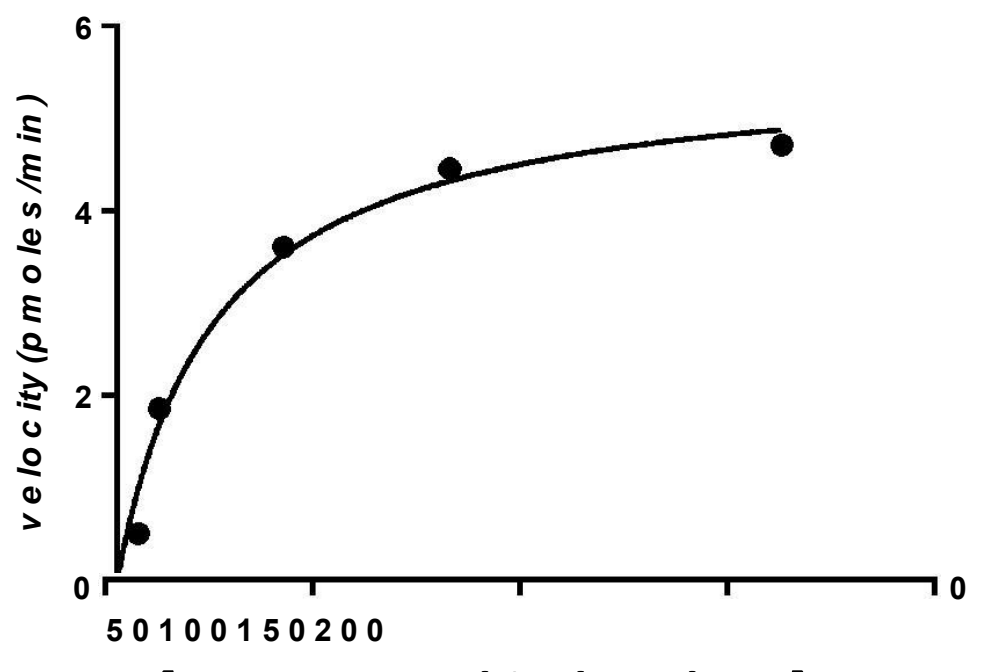

[Fructose-2,6-bisphosphate] $\mu \mathrm{M}$ 


\section{DISCUSSION}

Neoplastic transformation is characterized by an increase in glucose uptake and the conversion of glucose to lactate, even under aerobic conditions [2]. Glucose metabolism is regulated in part by the PFKFB family of enzymes. Of this family, the PFKFB3 and PFKFB4 enzymes have been found to be induced by hypoxia, co-expressed in several cancer cell lines, and function mainly to synthesize F-2,6-BP. A family of small molecule inhibitors targeting the kinase domain of PFKB3 (i.e. 3PO, PFK15, and PFK158) have been developed and in addition, a novel inhibitor of the PFKFB4 enzyme has recently been examined in vitro and in vivo and is under development. We sought to determine the effects of these inhibitors on the kinase activity of the PFKFB4 and PFKFB3 enzymes.

We first examined the effect of 5MPN on the kinase activity of PFKFB4. We found that 5MPN inhibits the kinase activity of PFKFB4 through competitive inhibition. In order to determine the specificity of the effect of 5MPN on PFKFB4, we also chose to examine its effect on the PFKFB3 enzyme, which is known to be highly expressed in multiple cancer cell types. We found when 5MPN was added to the kinase assay of PFKFB3 that no detectable inhibition was observed. These results suggest 5MPN specifically targets PFKFB4. 
Inhibitors of the PFKFB3 enzyme have been developed and a clinical candidate, PFK158, is currently being tested in human subjects in a Phase I trial [42]. With the known co-expression of PFKFB3 and PFKFB4 isoforms in multiple tumor types, we next sought to measure the effects of PFK15 and PFK158 on recombinant PFKFB4's kinase activity in comparison to its effect on PFKFB3. We found PFK15 and PFK158 competitively inhibit the kinase activity of both PFKFB4 and PFKFB3, however, both drugs inhibit PFKFB4 at a much lower efficiency than they do PFKFB3.

In order to characterize the interaction between the inhibitors and the enzymes, we then compared three different models of inhibition, competitive, non-competitive, and mixed. We found that all three small molecule inhibitors, PFK15, PFK158, and 5MPN, interacted with each isoform through competitive inhibition. However, we observed that the inhibitor designed to target the kinase domain of PFKFB4, 5MPN, had a minimal effect on the kinase activity of PFKFB3. Then, comparing $\mathrm{K}_{\mathrm{i}}$ values (see table below), we identified that PFK15 and PFK158 to be effective PFKFB3 inhibitors and 5MPN to be only effective at inhibiting the kinase activity of PFKFB4. Interestingly, we found that PFK15 was more effective than PFK158 at inhibiting PFKFB3 in vitro. 


\begin{tabular}{|l|l|c|c|}
\hline \multirow{2}{*}{} & & \multicolumn{2}{|c|}{$\mathrm{K}_{\mathbf{i}}$ VALUES } \\
\cline { 2 - 4 } $\begin{array}{l}\text { Small } \\
\text { molecule } \\
\text { Inhibitor }\end{array}$ & $\mathbf{5 M P N}$ & 8.6 & PFKFB4 \\
\cline { 2 - 4 } & PFK15 & 10.2 & N/A \\
\cline { 2 - 4 } & PFK158 & 20.9 & 3.26 \\
\hline
\end{tabular}

The Vmax increased to $19.5 \pm 1.0$ pmoles/minute from $11.7 \pm 0.8$ pmoles/minute and the $K_{\text {cat }}$ changed to $1.9 \pm 0.1 \mathrm{sec}^{-1}$ from $1.17 \pm 0.1$ $\sec ^{-1}$ We additionally found that inhibition of PFKFB4 by 5-MPN resulted in an increase in $V_{\max }$ of the bisphosphatase activity (to $19.5 \pm 1.0$ pmoles/second from $11.7 \pm 0.8$ pmoles/second) and changed the $\mathrm{K}_{\text {cat }}$ calculation to $1.9 \pm 0.1 \mathrm{sec}^{-1}$ from $1.17 \pm 0.1 \mathrm{sec}^{-1}$ We hypothesized that, due to the manner in which F-2,6-BP is synthesized, it is possible that contaminating ATP could be affecting the bisphosphatase reactions. If this were true, then the addition of kinase inhibitors should increase both the $V_{\text {max }}$ and $K_{\text {cat }}$ for both PFKFB3 and PFKFB4. We previously showed that PFK15 best inhibits PFKFB3 and 5-MPN is the most potent inhibitor of PFKFB4. Therefore, using these drugs in the bisphosphatase reaction should allow us to better isolate the bisphosphatase domains and more accurately analyze the bisphosphatase activities for both enzymes.

Without the inhibition of the kinase domain, then, in the presence of ATP, the reaction rate of the bisphosphatase domain appeared slower than it actually is. In fact, more accurate and higher bisphosphatase 
activity that was measured as a result caused the kinase to bisphosphatase ratio to drop from $4.1: 1$ as originally calculated to $2.7: 1$ (see table below).

\begin{tabular}{|l|c|c|}
\hline Isoform & PFKFB4 & PFKFB4 + \\
& & 5MPN \\
\hline Kinase Activity $\left(\mathrm{sec}^{-1}\right)$ & 5.03 & 5.03 \\
\hline Phosphatase Activity $\left(\mathrm{sec}^{-1}\right)$ & 1.17 & 1.9 \\
\hline Kinase:phosphatase ratio(sec & & \\
\hline
\end{tabular}

This phenomenon also held true for PFKFB3. Upon the addition of PFK15, the bisphosphatase reaction had a higher $V_{\max }$ and $K_{\text {cat. }}$ The $V_{\max }$ increased to $5.6 \pm 0.4$ from $4.3 \pm 0.1$ and $K_{\text {cat }}$ from $0.43 \pm 0.1 \mathrm{sec}^{-1}$ to .56 $\pm 0.04 \mathrm{sec}^{-1}$ (see table below). As a result, the activity ratio also decreased from 107:1 to 83:1. Although the activity ratio for PFKFB4 was lower than originally calculated, its kinase activity is still higher than its bisphosphatase activity which highlights its importance to developing cancer-specific therapies.

\begin{tabular}{|l|c|c|}
\hline Isoform & PFKFB3 & PFKFB3 + \\
& & PFK15 \\
\hline Kinase Activity $\left(\mathrm{sec}^{-1}\right)$ & 46.5 & 46.5 \\
\hline Phosphatase Activity $\left(\mathrm{sec}^{-1}\right)$ & .434 & .56 \\
\hline Kinase:phosphatase ratio $\left(\mathrm{sec}^{-1}\right)$ & $107: 1$ & $83: 1$ \\
\hline
\end{tabular}


Based on our observations, the small molecule inhibitors of PFKFB3 and PFKFB4 show selective effects against their targets. The PFKFB3 and PFKFB4 isoforms are co-expressed in a large number of tumor types and our findings suggest that combinations of inhibitors of these isoforms may prove to be an effective treatment to suppress tumor metabolism. 


\title{
CHAPTER VII
}

\author{
SUMMARY
}

Comparing and contrasting normal cells to cancer cells is important in identifying differences in cancer cell characteristics that can be potentially exploited. Inhibition of PFKFB3 has shown a lot of promise both in vivo and in vitro and a clinical trial using a PFKFB3 inhibitor, PFK158, has recently begun. However, because of its undetermined kinase to bisphosphatase ratio, PFKFB4 has been less studied. Recent data has demonstrated that PFKFB4 may play a more important role in cancer development than previously believed. Our study has shown biochemically that the kinase domain of PFKFB4 has greater activity than its bisphosphatase domain and, in addition, has demonstrated that a novel PFKFB4 inhibitor, 5MPN, specifically targets the PFKFB4 family member. Additionally, this study has shown evidence that the current PFKFB3 inhibitors are more specific to PFKFB3 than PFKFB4. Since PFKFB3 and PFKFB4 are co-expressed in a majority of cancers, the use of drugs to target both PFKFB3 and PFKFB4 in human cancers may prove to be a viable therapeutic strategy. 


\section{CHAPTER VIII}

\section{FUTURE DIRECTIONS}

\section{Understanding the effects of 5MPN in vitro and in vivo}

5MPN is a newly developed small molecule inhibitor of PFKFB4 and still under active investigation. We intend to further determine its efficacy in vivo and in vitro. We will examine the effects of 5MPN on normal cells in comparison to transformed cells and examine its effects on F-2,6-BP production, glucose uptake, glycolysis, and ATP production. Finally, we will perform in vivo toxicology studies and examine the effects of 5MPN on xenograft growth in mice.

\section{How other isoforms affected by PFKFB3 and PFKFB4 inhibitors}

Although PFKFB3 and now PFKFB4 have been examined in several studies, more research is necessary to fully understand how the members of this family of enzymes function and interrelate. In future studies, we intend to examine the effects of these small molecule inhibitors on PFKFB1 and PFKFB2.

Further examination of the bisphosphatase activites of the PFKFB3 and PFKFB4 enzymes 
Our initial results examining the bisphosphatase activity of both PFKFB3 and PFKFB4 yielded interesting results. However, a hurdle we have faced in the further examination of this activity has been a limited supply of F-2,6-BP, which is currently not available for purchase.

We intend to synthesize $\mathrm{F}-2,6-\mathrm{BP}$, in order to label it with ${ }^{32} \mathrm{Pi}_{\mathrm{i}}$ and then use scintillation to measure the production of product over time instead of the complicated enzyme coupled reaction we have relied on for these studies. This method would greatly simplify the analysis of the bisphosphatase activity of the members of this family of enzymes and allow for more accurate calculations. 


\section{REFERENCES}

[1] Hanahan, Douglas, and Robert A. Weinberg. "Hallmarks of Cancer: The Next Generation." Cell 144.5 (2011): 646-74.

[2] Warburg, O. "On the Origin of Cancer Cells." Science 123.3191 (1956): 309-14.

[3] Koppenol, Willem H., Patricia L. Bounds, and Chi V. Dang. "Otto Warburg's Contributions to Current Concepts of Cancer Metabolism." Nature Reviews Cancer 11.8 (2011): 618.

Boland, Michelle L., Aparajita H. Chourasia, and Kay F. Macleod. "Mitochondrial Dysfunction in Cancer." Frontiers in Oncology 3 (2013): 3:292.

[5] Heiden, M. G. Vander, L. C. Cantley, and C. B. Thompson. "Understanding the Warburg Effect: The Metabolic Requirements of Cell Proliferation." Science 324.5930 (2009): 1029-033.

[6] Lopez-Lazaro, Miguel. "The Warburg Effect: Why and How Do Cancer Cells Activate Glycolysis in the Presence of Oxygen?" Anti-Cancer Agents in Medicinal Chemistry (Formerly Current Medicinal Chemistry - AntiCancer Agents) 8.3 (2008): 305-12.

[7] Yalcin, Abdullah, Sucheta Telang, Brian Clem, and Jason Chesney. "Regulation of Glucose Metabolism by 6-phosphofructo-2-kinase/fructose2,6-bisphosphatases in Cancer." Experimental and Molecular Pathology 86.3 (2009): 174-79.

[8] Weber, George. "Enzymology of Cancer Cells." New England Journal of Medicine 296.10 (1977): 541-51.

[9] Rider, Mark H., Luc Bertrand, Didier Vertommen, Paul A. Michels, Guy G. Rousseau, and Louis Hue. "6-Phosphofructo-2-kinase/fructose-2,6bisphosphatase: Head-to-head with a Bifunctional Enzyme That Controls Glycolysis." Biochemical Journal 381.3 (2004): 561.

[10] Kountz, Paul D., M.raafat El-Maghrabi, and Simon J. Pilkis. "Isolation and Characterization of 6-phosphofructo-2-kinase/fructose-2,6- 
bisphosphatase from Bovine Liver." Archives of Biochemistry and Biophysics 238.2 (1985): 531-43.

[11] Kitamura, K and Uyeda, K. "The Mechanism of Activation of Heart Fructose-6-phosphate/fructose-2,6-bisphosphatase." Journal of Biological Chemistry (1987): 679-681.

[12] Ros, Susana, and Almut Schulze. "Balancing Glycolytic Flux: The Role of 6-phosphofructo-2-kinase/fructose 2,6-bisphosphatases in Cancer Metabolism." Cancer \& Metabolism 1.1 (2013): 8.

[13] Sakakibara, R., M. Kato, N. Okamura, T. Nakagawa, Y. Komada, N. Tominaga, M. Shimojo, and M. Fukasawa. "Characterization of a Human Placental Fructose-6-Phosphate, 2-Kinase/Fructose- 2,6 Bisphosphatase." Journal of Biochemistry 122.1 (1997): 122-28.

[14] Bobarykina, Anastasia, Minchenko Dmytro, Opentanova Iryna, Moenner Michel, Caro Jaime, Esumi Hiroyasu, and Minchenko Oleksandr. "Hypoxic regulation of PFKFB-3 and PFKFB-4 gene expression in gastric and pancreatic cancer cell lines and expression of PFKFB genes in gastric cancers." ACTA Biochimica Polinica; (2006): 789-799.

[15] Minchenko, Oleksandr, Iryna Opentanova, and Jaime Caro. "Hypoxic Regulation of the 6-phosphofructo-2-kinase/fructose-2,6-bisphosphatase Gene Family (PFKFB-1-4) Expression in Vivo." FEBS Letters 554.3 (2003): 264-70.

[16] Bando $H$, Atsumi $T$, Nishio $T$, Niwa $H$, Mishima $S$, Shimizu C, Yoshioka N, Bucala R, Koike T. "Phosphorylation of the 6-phosphofructo2-kinase/fructose 2,6- bisphosphatase/PFKFB3 Family of Glycolytic Regulators in Human Cancer." Clinical Cancer Research (2005). 11: 5784-5792.

[17] Minchenko, O.h., A. Ochiai, I.I. Opentanova, T. Ogura, D.o. Minchenko, J. Caro, S.v. Komisarenko, and H. Esumi. "Overexpression of 6-phosphofructo-2-kinase/fructose-2,6-bisphosphatase-4 in the Human Breast and Colon Malignant Tumors." Biochimie 87.11 (2005): 1005-010.

[18] Goidts, V., J. Bageritz, L. Puccio, S. Nakata, M. Zapatka, S. Barbus, G. Toedt, B. Campos, A. Korshunov, S. Momma, E. Van Schaftingen, G. Reifenberger, C. Herold-Mende, P. Lichter, and B. Radlwimmer. "RNAi Screening in Glioma Stem-like Cells Identifies PFKFB4 as a Key Molecule Important for Cancer Cell Survival." Oncogene (2011): 3235-3243.

[19] Ros, S., C. R. Santos, S. Moco, F. Baenke, G. Kelly, M. Howell, N. Zamboni, and A. Schulze. "Functional Metabolic Screen Identifies 6Phosphofructo-2-Kinase/Fructose-2,6-Biphosphatase 4 as an Important 
Regulator of Prostate Cancer Cell Survival." Cancer Discovery 2.4 (2012): $328-43$.

[20] Chesney, J., Clark, J., Klarer, A., Imbert-Fernandez, Y., Lane, A., \& Telang, S. "Fructose-2,6-Bisphosphate Synthesis by 6-Phosphofructo-2Kinase/Fructose-2,6- bisphosphatase 4 (PFKFB4) is Required for the Glycolytic Response to Hypoxia and Tumor Growth. Oncotarget (2014), 5(16), 6670-6686.

[21] Okar, David A., Alex J. Lange, Ànna Manzano, Aurèa NavarroSabatè, lu s Riera, and Ramon Bartrons. "PFK-2/FBPase-2: Maker and Breaker of the Essential Biofactor Fructose-2,6-bisphosphate." Trends in Biochemical Sciences 26.1 (2001): 30-35.

[22] Sakai A, Kato M, Fukasawa M. "Cloning of cDNA Encoding for a Novel Isoenzyme of Fructose-6-phosphate,2-kinase/fructose-2,6bisphosphatase From Human Placenta. Journal of Biochemistry (Tokyo) (1996). 119-505-511.

[23] Sakata J, Abe Y and Uyeda K. "Molecular Cloning of the DNA and Expression and Characterization of Rat Ttestes Fructose-6-phosphate,2kinase:fructose-2,6- bisphosphatase. Journal of Biological Chemistry (1991). 266(24): 15764-15770.

[24] Manzano, A., J.x Pérez, M. Nadal, X. Estivill, A. Lange, and R. Bartrons. "Cloning, Expression and Chromosomal Localization of a Human Testis 6-phosphofructo-2-kinase/fructose-2,6-bisphosphatase Gene." Gene 229.1-2 (1999): 83-89

[25] Schaftingen, E. Van. "Fructose 2,6-bisphosphate, a Glycolytic Signal." Archives Of Physiology And Biochemistry 94.2 (1986): 151-59.

[26] Hue L., Rider M. H. "Role of Fructose 2,6-bisphosphate in the Control of Glycolysis in Mammalian Tissues." Journal of Biochemistry (1987). 245:313-324.

[27] Pilkis, S. "6-Phosphofructo-2-Kinase/Fructose-2,6-Bisphosphatase: A Metabolic Signaling Enzyme." Annual Review of Biochemistry 64.1 (1995): 799-835.

[28] Kim, S.G. "Crystal Structure of the Hypoxia-inducible Form of 6Phosphofructo-2-kinase/fructose-2,6-bisphosphatase (PFKFB3): A POSSIBLE NEW TARGET FOR CANCER THERAPY." Journal of Biological Chemistry 281.5 (2005): 2939-944. 
[29] Michels, Paul, and Daniel Rigden. "Evolutionary Analysis of Fructose 2,6-bisphosphate Metabolism." IUBMB Life (International Union of Biochemistry and Molecular Biology: Life) 58.3 (2006): 133-41.

[30] Lee, Y.-H. "Tissue-specific Structure/Function Differentiation of the Liver Isoform of 6-Phosphofructo-2-kinase/Fructose-2,6-bisphosphatase." Journal of Biological Chemistry 278.1 (2002): 523-30.

[31] Hasemann, Charles A., Eva S. Istvan, Kosaku Uyeda, and Johann Deisenhofer. "The Crystal Structure of the Bifunctional Enzyme 6phosphofructo-2-kinase/fructose-2,6-bisphosphatase Reveals Distinct Domain Homologies." Structure 4.9 (1996): 1017-029.

[32] Sakata J, Abe Y and Uyeda K. "Molecular Cloning of the DNA and Expression and Characterization of Rat Testes Fructose-6-phosphate,2kinase:fructose-2,6- bisphosphatase." Journal of Biological Chemistry (1991). 266(24):15764-15770.

[33] Schaftingen, Emile, Beatrice Lederer, Ramon Bartrons, and HenriGery Hers. "A Kinetic Study of Pyrophosphate: Fructose-6-Phosphate Phosphotransferase from Potato Tubers. Application to a Microassay of Fructose 2,6-Bisphosphate." European Journal of Biochemistry 129.1 (1982): 191-95

[34] Sakakibara, R., M. Kato, N. Okamura, T. Nakagawa, Y. Komada, N. Tominaga, M. Shimojo, and M. Fukasawa. "Characterization of a Human Placental Fructose-6-Phosphate, 2-Kinase/Fructose- 2,6 Bisphosphatase." Journal of Biochemistry 122.1 (1997): 122-28.

[35] Pilkis SJ, Regen DM, Stewart HB, Pilkis J, Pate TM, and El-Maghrabi MR. " Evidence for Two Catalytic Sites on 6-phosphofructo-2kinase/fructose 2,6- bisphosphatase. Dynamics of Substrate Exchange and Phosphoryl Enzyme Formation." Journal of Biological Chemistry (1984); .259:949-957.

[36] Tauler A, Lin K and Pilkis SJ. "Hepatic 6-phosphofructo-2kinase/fructose-2,6- bisphosphatase. Use of Site-directed Mutagenesis to Evaluate the Roles of His-258 and His-392 in Catalysis." Journal of Biological Chemistry (1990). 265:15617-15622

[37] Obach, M., A. Navarro-Sabate, J. Caro, X. Kong, J. Duran, M. Gomez, J. C. Perales, F. Ventura, J. L. Rosa, and R. Bartrons. "6Phosphofructo-2-kinase (pfkfb3) Gene Promoter Contains Hypoxiainducible Factor-1 Binding Sites Necessary for Transactivation in Response to Hypoxia." Journal of Biological Chemistry 279.51 (2004): 53562-3570. 
[38] Lin K, Li L, Correia J and Pilkis SJ. " Arg-257 and Arg-307 of 6phosphofructo-2-kinase/fructose-2,6- bisphosphatase Bind the C-2 phospho Group of Fructose-2,6-bisphosphate in the Fructose-2,6bisphosphatase Domain." Journal of Biological Chemistry (1992). 267; 19163-19171

[39] Telang, S., A. Yalcin, A. L. Clem, R. Bucala, A. N. Lane, J. W. Eaton, and J. Chesney. "Ras Transformation Requires Metabolic Control by 6phosphofructo-2-kinase." Oncogene 25.55 (2006): 7225-234.

[40] Atsumi T, Chesney J, Metz C, Leng L, Donnelly S, Makita Z, Mitchell $\mathrm{R}$, Bucala R. "High Expression of inducible 6-phosphofructo-2kinase/fructose-2,6- bisphosphatase (iPFK-2; PFKFB3) in human cancers." Cancer Research (2002). 62:5881-7.

[41] Clem, B. F., J. O'neal, G. Tapolsky, A. L. Clem, Y. Imbert-Fernandez, D. A. Kerr, A. C. Klarer, R. Redman, D. M. Miller, J. O. Trent, S. Telang, and J. Chesney. "Targeting 6-Phosphofructo-2-Kinase (PFKFB3) as a Therapeutic Strategy against Cancer." Molecular Cancer Therapeutics 12.8 (2013): 1461-470.

[42] Telang S, O'Neal J, Tapolsky G, Clem B, Ker A, Imbert-Fernandez Y, Chesney J. "Discovery of a PFKFB3 inhibitor for phase I trial testing that synergizes with the B-Raf inhibitor vemurafenib." Cancer and Metabolism (2014). 2(Suppl 1):P14

[44] Dick Mitchell. Help Topics for Exploratory Enzyme Kinetics. Sigmaplot. Systat, Inc. Web.

[45] Orphardt, Charles . "Vitural Chembook." Elmhurst College, 2009.

[46] Mohan, Chandra, Kevin D. Long, and Manpreet Mutneja. "An Introduction to Inhibitors and Their Biological." EMB Millipore, 2013.

[47] Beers, Melissa. "The Michaelis-Menten Model." Wellesley College, 2010.

[48] Cohen JS, Lyon RC, Chen C, Faustino PJ, Batist G, Shoemaker M, Rubalcaba $\mathrm{E}$ and Cowan $\mathrm{KH}$. Differences in phosphate metabolite levels in drug-sensitive and -resistant human breast cancer cell lines determined by 31P magnetic resonance spectroscopy. Cancer Res. 1986; 46(8):40874090.

[49] Imbert-Fernandez Y, Clem BF, O'Neal J, Kerr DA, Spaulding R, Lanceta L, Clem AL, Telang $S$ and Chesney J. Estradiol stimulates glucose metabolism via 6-phosphofructo-2-kinase (PFKFB3). J Biol Chem. 2014; 289(13):9440-9448. 


\section{CURRICULUM VITAE}

Name: Jennifer Amanda Clark

\section{Education \& Training:}

Institution

Franklin

College

Franklin,

Indiana

Research and Job Experience:

$\begin{array}{llll}\begin{array}{l}\text { Position/Title } \\ \text { Associate }\end{array} \text { Scientist } & \text { Research } & \begin{array}{l}\text { Year } \\ 2009-\end{array} & \begin{array}{l}\text { Employer/Location } \\ \text { Apolmmune } \\ \text { Louisville, Ky } \\ \text { University of Louisville } \\ \text { Department of Medicine } \\ \text { Louisville, Ky }\end{array} \\ \text { Research Technician IV } & \begin{array}{l}2010 \\ 2010-\end{array} & \begin{array}{l}\text { Mississippi Department of Public } \\ \text { Research Technician IV }\end{array} & \begin{array}{l}\text { 2014- } \\ \text { Present } \\ \text { Dealth } \\ \text { transmitted Disease } \\ \text { Jackson, Mississippi }\end{array}\end{array}$

Field of Study

Chemistry/Biology $\begin{array}{ll}\text { Degree } & \text { Years } \\ \text { B.A. } & 2005 \\ & 2009\end{array}$ 
Donninger, H., N. Allen, A. Henson, J. Pogue, A. Williams, L. Gordon, S. Kassler, T. Dunwell, F. Latif, and G. J. Clark. "Salvador Protein Is a Tumor Suppressor Effector of RASSF1A with Hippo Pathway-independent Functions." Journal of Biological Chemistry 286.21 (2011): 18483-8491.

Donninger, Howard, Thibaut Barnoud, Nick Nelson, Suzanna Kassler, Jennifer Clark, Timothy D. Cummins, David W. Powell, Sarah Nyante, Robert C. Millikan, and Geoffrey J. Clark. "RASSF1A and the Rs2073498 Cancer Associated SNP." Frontiers in Oncology 1 (2011)

Clark, Jennifer, Jessica Freeman, and Howard Donninger. "Loss of RASSF2 Enhances Tumorigencity of Lung Cancer Cells and Confers Resistance to Chemotherapy." Molecular Biology International 2012 (2012): 1-8.

Klarer, Alden C., Julie O'Neal, Yoannis Imbert-Fernandez, Amy Clem, Steve R. Ellis, Jennifer Clark, Brian Clem, Jason Chesney, and Sucheta Telang. "Inhibition of 6-phosphofructo-2-kinase (PFKFB3) Induces Autophagy as a Survival Mechanism." Cancer \& Metabolism 2.1 (2014): 2

Chesney, J., Clark, J., Klarer, A., Imbert-Fernandez, Y., Lane, A., \& Telang, S. (2014). Fructose-2,6-Bisphosphate synthesis by 6Phosphofructo-2-Kinase/Fructose-2,6- bisphosphatase 4 (PFKFB4) is required for the glycolytic response to hypoxia and tumor growth. Oncotarget, 5(16), 6670-6686.

Donninger, H., Clark, J., Rinaldo, F., Nelson N., Barnoud T., Schmidt, ML., Hobbing, KR., Vos, MD., Sills, B., and Clark GJ. "The RASSF1A Tumor Suprressor Regulates XPA-Mediated DNA Repair." Molecular Cell Biology (2014): 00202-14.

\section{National Meeting Presentations:}

Metabolism, Diet and Disease Conference: Cancer and MetabolismPoster Presentation:

J. Clark, A. Lane, J. Chesney, S. Telang. "The Kinase Domain of PFKFB4 is Required to Stimulate Glucose Metabolism and Growth in H460 Lung Adenocarcinoma Cells." 